\title{
Sources of CAM3 vorticity bias during northern winter from diagnostic study of the vorticity equation
}

\author{
Richard Grotjahn • Lin-Lin Pan · Joseph Tribbia
}

Received: 15 October 2009/Accepted: 17 January 2011/Published online: 3 February 2011

(c) The Author(s) 2011. This article is published with open access at Springerlink.com

\begin{abstract}
CAM3 (Community Atmosphere Model version 3) simulation bias is diagnosed using the vorticity equation. The study compares CAM3 output with ECMWF (European Centre for Medium-Range Weather Forecasts) 40 year reanalysis (ERA-40) data. A time mean vorticity bias equation is also formulated and the terms are grouped into categories: linear terms, nonlinear terms, transient contributions, and friction (calculated as a residual). Frontal cyclone storms have much weaker band passed kinetic energy and enstrophy in CAM3. The downstream end of the North Atlantic storm track (NAST) has large location error. While the vorticity equation terms have similar amplitude ranking in CAM3 and ERA-40 at upper levels, the ranking differs notably in the lower troposphere. The linear and friction terms dominate the vorticity bias equation. The transient terms contribute along the storm track, but the nonlinear terms are generally much smaller, with the primary exception being over the Iberian peninsula. Friction is much stronger in CAM3. As evidence, nearly all wavelengths (including the longest planetary waves) have smaller amplitude in CAM3 than in ERA-40 vorticity data. Negative near surface vorticity tendency bias on the European side of the Arctic is linked to the NAST track error (evident in the divergence term). CAM3 misses the Beaufort high in sea level pressure (SLP) due to low level warm temperature bias, too little vortex compression, and to too little horizontal advection of negative
\end{abstract}

R. Grotjahn $(\bowtie)$

Department of Land, Air and Water Resources,

University of California, Davis, CA 95616, USA

e-mail: grotjahn@ucdavis.edu

L.-L. Pan · J. Tribbia

National Center for Atmospheric Research, Boulder, CO, USA vorticity compared with ERA-40. Generally lower SLP values in CAM3 over the entire Arctic follow from lower level warm bias in CAM3.

Keywords CAM3 vorticity bias - Vorticity equation . Climate model bias - Northern hemisphere storm tracks . Arctic climate

\section{Introduction}

The primary purpose of this article is to advance understanding of the bias in the rotational part of the wind fields simulated by the National Center for Atmospheric Research (NCAR) community atmosphere model version 3 (CAM3). In this report, emphasis is upon the middle and higher latitudes of the Northern Hemisphere during boreal winter. The primary diagnostic tool is a vorticity bias equation, formed from the difference between the primitive vorticity equation using CAM3 versus the same equation using observational data. (Bias in any variable is defined as the model value minus the corresponding observed value of that variable). Bias in a vorticity equation term is found by subtracting the term using observation-based analysis data from the same term using CAM3 data.

This paper is a companion to an earlier paper (Pan et al. 2010; hereafter PGT) that examines an analogous temperature bias equation for CAM3. The equation in PGT is formed from the difference between a temperature equation using CAM3 data minus the same equation using observational data. The observational data chosen by PGT were the European Centre for Medium-Range Weather Forecasts (ECMWF) reanalysis dataset ERA-40. In PGT daily data were averaged over a 20 year period of December, January, and February (DJF). Being a long-term average, 
the tendency term could be neglected in the temperature bias equation. The remaining terms in the temperature bias equation were grouped into 4 categories: linear, nonlinear, transient, and diabatic terms. The linear terms are all those terms in which the model bias appears once in each term; these are horizontal and vertical advection terms by the bias flow of the observed temperature and by the observed winds of the temperature bias. The nonlinear terms are of the bias flow advecting the bias temperature. The transient terms are time mean contributions by the transients to temperature advection in CAM3 minus the corresponding contributions from ERA-40 data. The diabatic terms include various forms of heating and cooling. In this study we formulate a corresponding vorticity bias equation: by subtracting the vorticity equation terms using ERA-40 data from the same terms using CAM3 data. We also make a similar partitioning into linear, transient, nonlinear, and instead of a diabatic term here we have a residual term that includes friction, diffusion, and unresolved processes.

The temperature and vorticity bias equations have parallels to the equations used by linear stationary wave (LSW) models (e.g. Branstator 1990; Pan et al. 2006). The LSW model analog to the temperature bias equation treats the bias fields as a 'stationary wave'. Using an LSW model that way neglects the nonlinear terms (bias advecting bias), linear terms become a linear operator on the bias, and the transient and diabatic residual terms are treated as 'forcing' for the bias. The accuracy of an LSW model hinges upon whether the nonlinear terms can be neglected. PGT found that the temperature bias equation nonlinear terms were negligible most places (outside the deep tropics) and thus support using a LSW model to study the bias further, at least for the temperature equation. A LSW model also has divergence and vorticity (or horizontal velocity components) equations. The vorticity bias equation has a similar analog equation in the LSW linear operator. Hence, a second purpose of this report is to determine if the corresponding nonlinear terms for a vorticity bias equation can be similarly neglected.

In the tropics, PGT found large values for the linear and diabatic terms; PGT also found notable values for the transient and nonlinear terms near the intertropical convergence zones (ICZ). In middle and higher latitudes, PGT found that the transient, diabatic, and linear terms were larger in the midlatitude storm track regions. They found the temperature bias equation variation along the North Pacific storm track (NPST) to be quite different from how the terms vary along the North Atlantic storm track (NAST). The differences between these storm tracks were similar to different biases in the subtropical jets. Hence a third purpose of this article is to see if the NPST and NAST also have prominent roles in the vorticity bias equation and further explore the differences between the simulated NPST and NAST.
See PGT for a summary of some other aspects of the CAM3 bias, including how it changes with model resolution, and how it is similar to bias in the corresponding Community Climate System (CCSM3) coupled model. Our original interest in looking at the bias is to understand better why a similar bias appears in CAM3 and CCSM3 over the Arctic region. That bias in the surface winds creates significant errors in the sea ice simulation by CCSM3. The simulation of Arctic sea ice, air temperature and hydrology in some regions are also improved in the higher-resolution atmosphere (e.g. DeWeaver and Bitz 2006). On the other hand, some biases in the higher-resolution simulation may become more serious. Hack et al. (2006) conclude that the high-resolution version of the CAM3, especially the coupled model results do not offer unequivocal improvement. Since our original focus was upon the Arctic, this paper emphasizes the middle and high latitude vorticity bias equation results.

The CAM3 standard versions using a spectral formulation support three horizontal resolutions: triangular spectral truncations at 31,42 , or 85 zonal wavenumbers. CAM 3 uses 26 levels in the vertical with a hybrid terrain-following coordinate: sigma coordinates in the lowest layer, pressure at upper levels (approximately $83 \mathrm{hPa}$ or above), and hybrid sigma-pressure coordinates in between (Collins et al. 2004). The horizontal resolutions T42 and T85 are often used in CAM3 applications, and several studies (e.g. Hack et al. 2006) have investigated the differences in the simulation results between these two horizontal spectral truncations.

At most levels, including the surface winds, the Arctic surface climate bias in CAM3 is sufficiently similar to the bias in the coupled model (CCSM3) so that we assume that CAM3 is an adequate model to examine the primary sources of Arctic region bias in CCSM3. By studying CAM3, we avoid the complicating issues of biases in the ocean and sea ice models in CCSM3. Similarly, our focus is upon the winter months when variations in sea ice thickness develop.

The outline of the paper is as follows. The method used in this diagnostic study is briefly presented in the next section. Proxy measures of the northern hemisphere storm tracks (and corresponding bias) are discussed in Sect. 3. Section 4 shows the bias in various terms in the vorticity equation, including linear terms, nonlinear terms, transient, and frictional residual contributions to the time mean. The paper concludes with a summary discussion.

\section{Vorticity bias equation derivation}

A primary diagnostic used here is the vorticity bias equation. The equation is formed by evaluating the time mean vorticity equation using CAM 3 data then subtracting the 
same terms evaluated using observation-based data. The CAM3 data used here are obtained by running a 20 year AMIP (Atmospheric Model Intercomparison Project) type simulation from 1979 to 1998 . The model version used here includes all physics, has 26 levels in the vertical and the horizontal resolution is triangular truncation at wavenumber 42 (T42). The output is saved 4 times daily. Only the Northern Hemisphere winter months: December, January, and February are studied. The observational data used here are gridded $4 \times$ daily ERA-40 reanalysis data (Uppala et al. 2005) from 1979 to 1998 . The variables used here include zonal wind, meridional wind, and vertical velocity in $p$-coordinates.

The vorticity $(\zeta)$ equation in pressure $(p)$ coordinates is: $\frac{\partial \zeta}{\partial t}+\vec{V} \cdot \nabla(\zeta+f)+\omega \frac{\partial \zeta}{\partial p}+(\zeta+f) \nabla \cdot \vec{V}$

$-k \cdot\left(\frac{\partial \vec{V}}{\partial p} \times \nabla \omega\right)=F_{\zeta}$,

where $\vec{V}, \omega, \zeta, f, k$, and $F_{\zeta}$ denote horizontal wind vector, vertical velocity in $p$ coordinates, vertical component of relative vorticity, Coriolis parameter, vertical direction unit vector, and friction residual respectively. We evaluate the vorticity equation in pressure coordinates since ERA-40 and CAM3 data are both available at such levels. (If hybrid
We indicate time averaging with an overbar and use a prime notation for the deviation from that average. Subscript " $C$ " denotes CAM3 data; subscript " $E$ " denotes ERA-40 data. For the time mean of the CAM3 model output we have:

$$
\begin{aligned}
& \overline{\vec{V}_{C}} \cdot \nabla\left(\overline{\zeta_{C}}+f\right)+\overline{\omega_{C}} \frac{\partial \overline{\zeta_{C}}}{\partial p}+\left(\overline{\zeta_{C}}+f\right) \nabla \cdot \overline{\vec{V}_{C}}-\vec{k} \cdot\left(\frac{\partial \vec{V}_{C}}{\partial p} \times \nabla \overline{\omega_{C}}\right)
\end{aligned}
$$

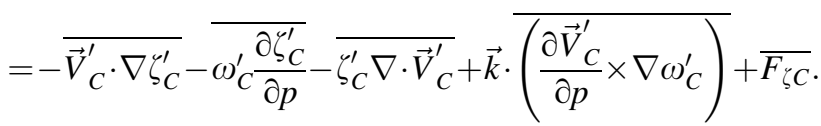

For the time mean of the ERA-40 observational data we have:

$$
\begin{gathered}
\overline{\vec{V}}_{E} \cdot \nabla\left(\overline{\zeta_{E}}+f\right)+\overline{\omega_{E}} \frac{\partial \overline{\zeta_{E}}}{\partial p}+\left(\overline{\zeta_{E}}+f\right) \nabla \cdot \overline{\vec{V}_{E}}-\vec{k} \cdot\left(\frac{\partial \overrightarrow{\vec{V}}_{E}}{\partial p} \times \nabla \overline{\omega_{E}}\right) \\
=-\overline{\vec{V}_{E}^{\prime} \cdot \nabla \zeta_{E}^{\prime}}-\overline{\omega_{E}^{\prime} \frac{\partial \zeta_{E}^{\prime}}{\partial p}}-\overline{\zeta_{E}^{\prime} \nabla \cdot \vec{V}_{E}^{\prime}}+\vec{k} \cdot \overline{\left(\frac{\partial \vec{V}_{E}^{\prime}}{\partial p} \times \nabla \omega_{E}^{\prime}\right)}+\overline{F_{\zeta E}} .
\end{gathered}
$$

$\mathrm{A} \wedge$ notation indicates the bias, for example: $\bar{V}_{C}-\bar{V}_{E}=\hat{V}$. Subtracting Eqs. 2-3 yields:

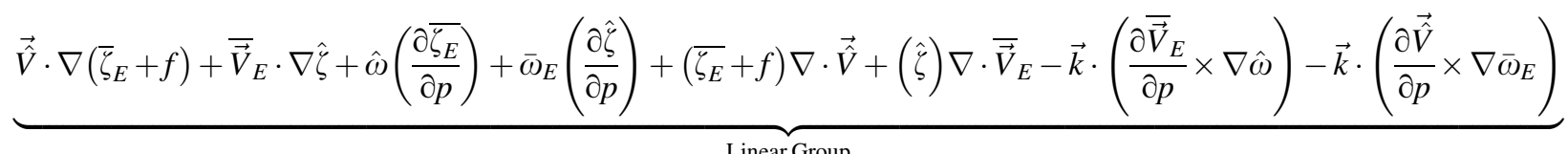

$$
\begin{aligned}
& =\underbrace{-\overrightarrow{\hat{V}} \cdot \nabla \hat{\zeta}-\hat{\omega}\left(\frac{\partial \hat{\zeta}}{\partial p}\right)-\hat{\zeta} \nabla \cdot \overrightarrow{\hat{V}}+\vec{k} \cdot\left(\frac{\partial \overrightarrow{\hat{V}}}{\partial p} \times \nabla \hat{\omega}\right)}_{\text {Nonlinear Group }} \\
& \underbrace{-\overline{\vec{V}_{C}^{\prime} \cdot \nabla \zeta_{C}^{\prime}}-\overline{\omega_{C}^{\prime}\left(\frac{\partial \zeta_{C}^{\prime}}{\partial p}\right)}-\overline{\zeta_{C}^{\prime} \nabla \cdot \vec{V}_{C}^{\prime}}+\vec{k} \cdot \overline{\left(\frac{\partial \vec{V}_{C}^{\prime}}{\partial p} \times \nabla \omega_{C}^{\prime}\right)}+\overline{\vec{V}_{E}^{\prime} \cdot \nabla \zeta_{E}^{\prime}}+\overline{\omega_{E}^{\prime}\left(\frac{\partial \zeta_{E}^{\prime}}{\partial p}\right)}+\overline{\zeta_{E}^{\prime} \nabla \cdot \vec{V}_{E}^{\prime}}-\vec{k} \cdot \overline{\left(\frac{\partial \vec{V}_{E}^{\prime}}{\partial p} \times \nabla \omega_{E}^{\prime}\right)}}_{\text {Transient Group }}+\hat{F}_{\zeta} .
\end{aligned}
$$

or terrain following coordinates native to the datasets were used, the levels would not match. Also, topographic elevation specification differs notably between the two datasets. However, the results are transformed to the terrain following coordinates of the LSW model when plotted in this paper. Doing so matches our prior study, PGT, and so facilitates comparison with our earlier paper. It also assists the purpose of evaluating future use of the LSW model.)
The terms at the left hand side are all terms that are linear in the bias; the aggregate of these terms is referred to as the Linear Group. These terms are 'linearized' about the time mean observed flow. Hence, the terms in the Linear Group would be present in a linear stationary wave (LSW) model's linear operator (the terms form the linear operator on the vorticity bias). The first 4 terms on the right hand side (labeled Nonlinear Group) are all nonlinear combinations of 
the bias. The group of terms labeled Transient Group has all transient contributions to the vorticity bias equation; it is the difference between the transient contributions to the time mean terms using ERA-40 and CAM3 data.

The term $\hat{F}_{\zeta}$ is intended to be the bias in diffusion and friction and is evaluated as a residual. In practice, there can be several contributions to this term from the numerics of the models used, such as diffusion implicit in the numerical scheme (particularly for the vertical derivatives) and unresolved processes. Because the spectrum of vorticity advection does not fall off very quickly, this residual term can have notable contributions from interacting high wavenumbers beyond the CAM3 model resolution that feed vorticity back onto resolved scales. The problem is worsened by using a smaller (T42) truncation than the truncation (T63) used to generate the ERA-40 reanalysis data.

\section{Bias in northern hemisphere storm tracks}

In PGT a proxy for the midlatitude storm track was the time mean of the transient meridional heat flux, $\overline{v^{\prime} T^{\prime}}$. The transients were defined by band-pass filtering the data to allow 2-8 day period fluctuations of meridional wind component (v) and temperature $(T)$. PGT discuss the bias of this heat flux. In this study two other proxy indicators of the storm track are shown: time means of kinetic energy and enstrophy (vorticity squared) from band-passed (2-8 day) filtered transient winds. The former is abbreviated $\mathrm{KE}^{\prime}$ and the latter Ens' hereafter. The transient meridional heat flux $\left(v^{\prime} T^{\prime}\right)$ is known to emphasize the early and mature stages of frontal cyclones (e.g. Grotjahn 1993). In contrast, $\mathrm{KE}^{\prime}$ and Ens' tend to emphasize the later stages of the frontal systems. The storm track is identified here as the latitudes where the proxy variable has maximum value at each longitude along the track. Representative results for $\mathrm{KE}^{\prime}$ and Ens' are shown in Fig. 1 where time mean patterns are plotted at $\sigma=0.3$, approximately the $300 \mathrm{hPa}$ level, for ERA-40 data, CAM3 data, and their difference (Ens' and $\mathrm{KE}^{\prime}$ biases).

The overall impressions of the storm track bias are: The model does produce both a north Atlantic storm track (hereafter NAST) and a separate north Pacific storm track (NPST), but the storm track proxies, $\mathrm{KE}^{\prime}$ and Ens', both have much smaller magnitude in CAM3 than in ERA-40. Peak values along the NAST are nearly 3 times as large for Ens' and about a third larger for $\mathrm{KE}^{\prime}$ in ERA-40. It is beyond the scope of this study to explain this difference. However, the following information may be relevant. The resolution used to generate the ERA-40 data (T63) is larger than the resolution used to generate the CAM3 data (T42). The ERA-40 data are truncated spectrally to the CAM3 resolution before the two datasets are interpolated to the
Fig. 1 High pass (2-8 day periods) wind-related products: transient kinetic energy ( $\mathrm{KE}^{\prime}$, left column, in $\mathrm{m}^{2} \mathrm{~s}^{-2}$ ) and transient enstrophy (vorticity squared, Ens', right column, in $\mathrm{s}^{-2}$ ) during DJF conditions. The level is $\sigma=0.3$. Top row: 1979-1998 DJF time averages for ERA-40. Middle row: DJF averages for 20 year CAM3 simulation using historical boundary conditions. Bottom row: $\mathrm{KE}^{\prime}$ and Ens' bias: values in middle row minus corresponding values in top row. The contour interval is uniform within a column. Along the storm tracks $\mathrm{KE}^{\prime}$ in CAM3 is about $3 / 4$ the corresponding value in ERA-40; for Ens ${ }^{\prime}$ the CAM3 values are about a third the corresponding ERA-40 values

same grid before making calculations shown here, so the final resolutions used to calculate the storm track proxies and the bias match. Perhaps there may be different energy and enstrophy cascades occurring due to the difference in resolution used in the original datasets. A wavelet analysis is applied to the transient relative vorticity and meridional wind fields to see if those fields differ in scale between CAM3 and ERA-40. Transient $\zeta$ and $v$ fields are used since they have positive and negative values that relate to the scale of the weather systems in the storm track. Wavelet transforms, using the DOG (derivative of Gaussian) wavelet, are applied to daily maps, then the wavelet magnitudes are averaged in time. Zonal wavelet transforms of vorticity are shown in Fig. 2b, c. The wavelet analysis finds that the wavenumbers having largest amplitude are somewhat longer for CAM3 than ERA-40. The peak vorticity in the NAST (at $40^{\circ} \mathrm{N}$ and $45^{\circ} \mathrm{N}$ ) occurs between wavenumbers 6 and 7 in CAM3 and close to wavenumber 7 in ERA-40. For the NPST, the peak wavenumber has the same scale in CAM3 and ERA-40. For both the NAST and NPST, the CAM3 magnitudes of $\mathrm{KE}^{\prime}$ and Ens' are much less for all wavenumbers. CAM3 values are roughly half the corresponding values in ERA-40 at all wavenumbers and locations at or near the storm tracks. Spectral transforms (Fig. 2a) along midlatitude circles find nearly all of the longest waves (wavenumbers 1-10) have less amplitude in CAM3 than in ERA-40. Hence, the greater enstrophy in ERA-40 is not explained by higher amplitude short waves alone because nearly all waves have higher amplitude in ERA-40. Perhaps the diminished amplitude simply reflects the generally greater extraction of energy and amplitude in CAM3 than in ERA-40 by friction and diffusion. The vorticity bias equation friction and diffusion group of terms, shown later, is generally larger along the NAST and NPST in CAM3, especially at lower levels. Finally, ERA-40 data and CAM3 simulations both have a much stronger NAST than NPST.

Grotjahn and Castello (2000) examined $300 \mathrm{hPa}$ level geostrophic kinetic energy anomaly (with a sector average removed) and found the scale increased as storms developed along the NPST. The wavelet analysis here finds a 


\section{At $\operatorname{sigma}=0.3$}

a

ERA-40 (U'U'+V'V')/2.

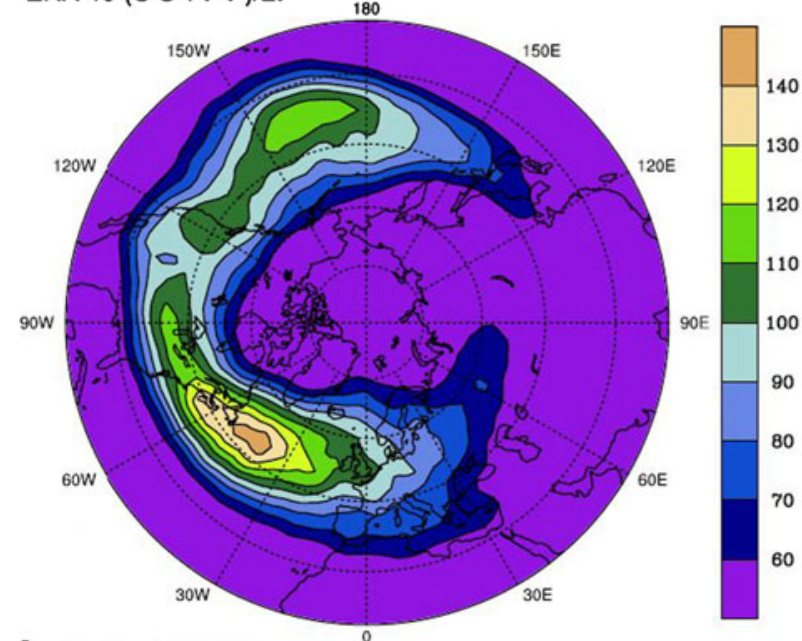

b

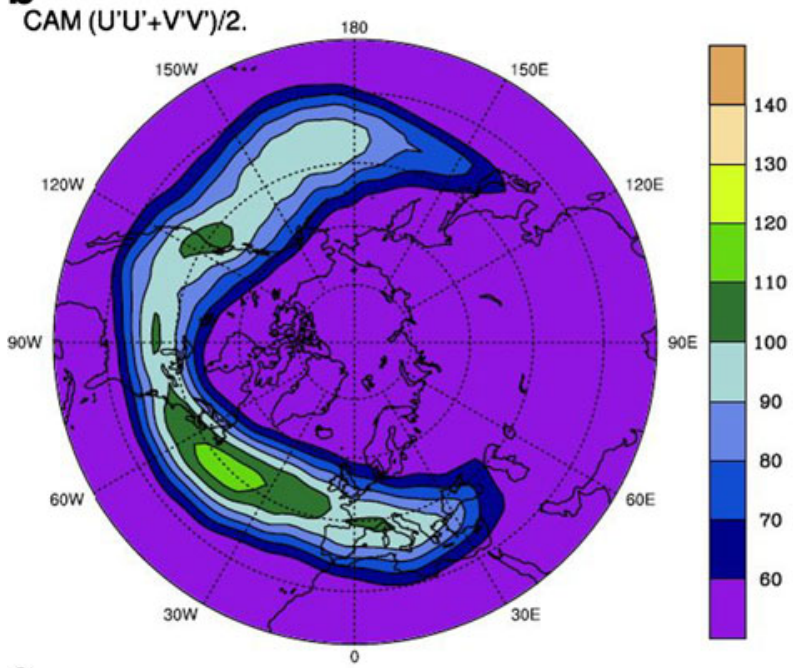

C

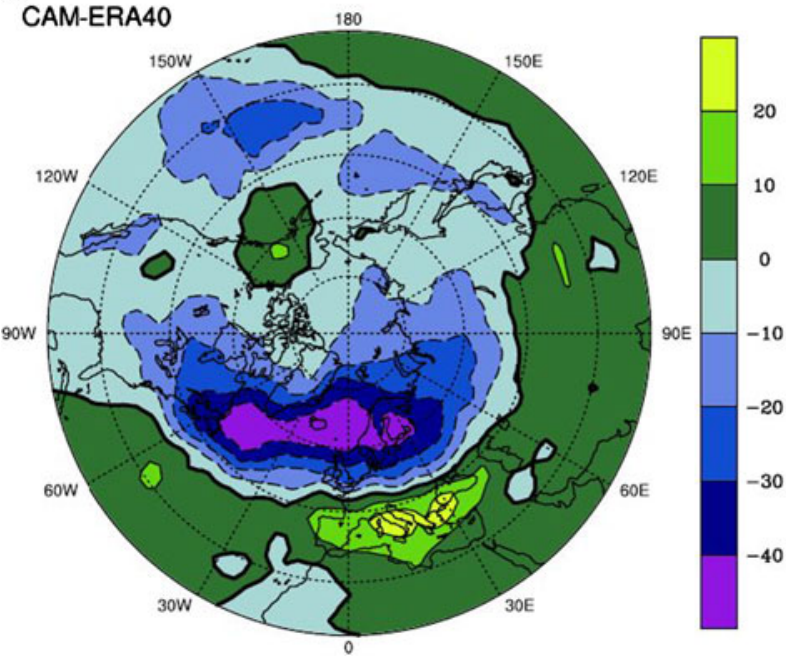

d

At sigma $=0.3$

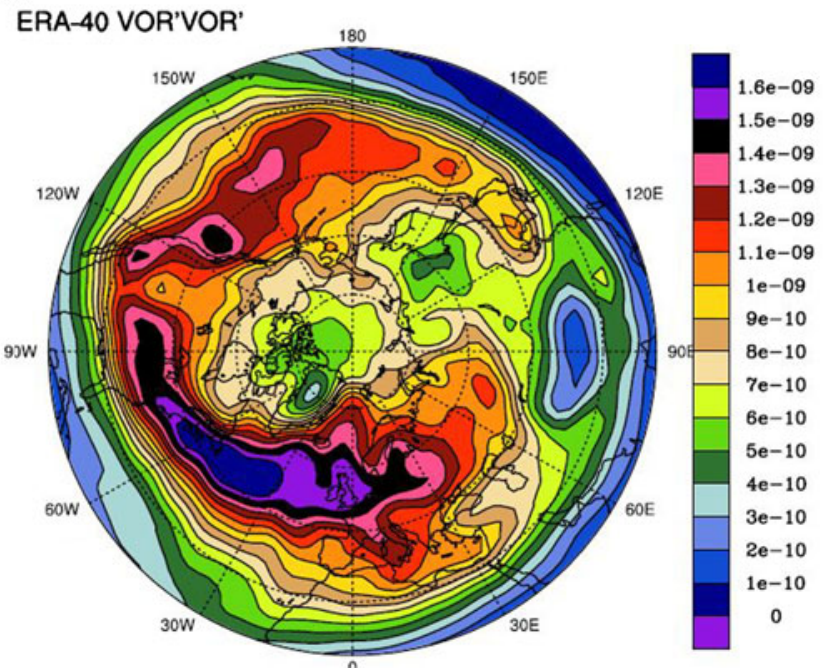

e

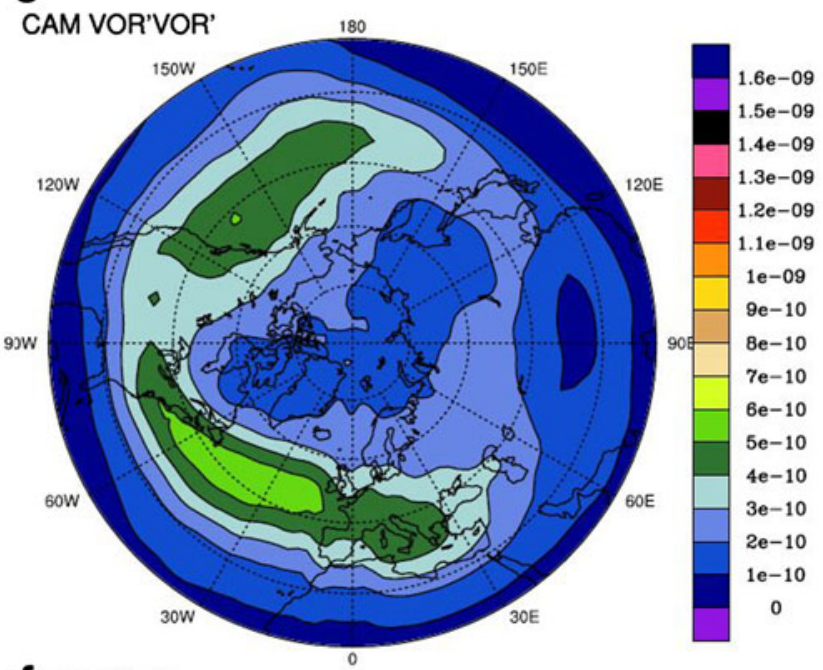

$f_{\text {CAM-ERA40 }}$

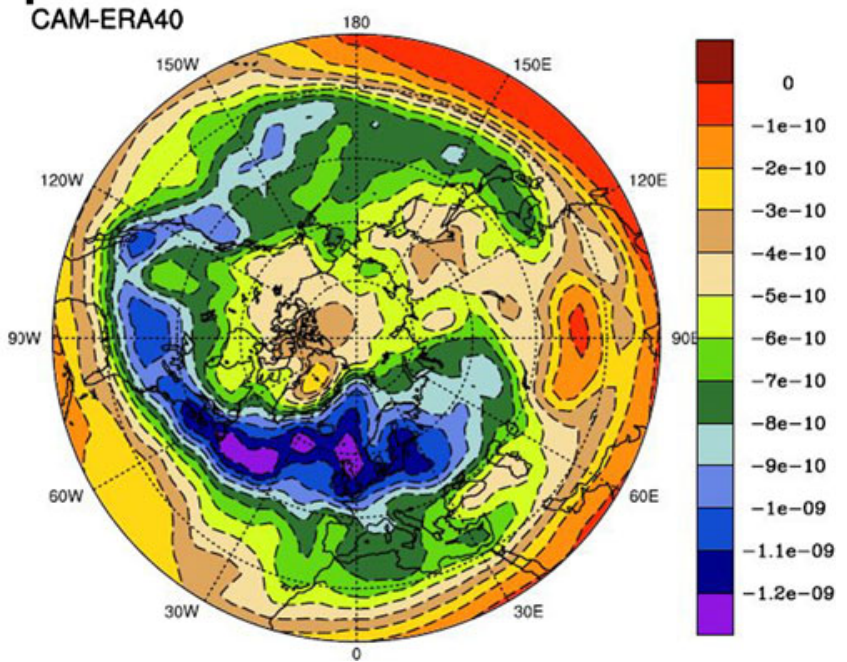



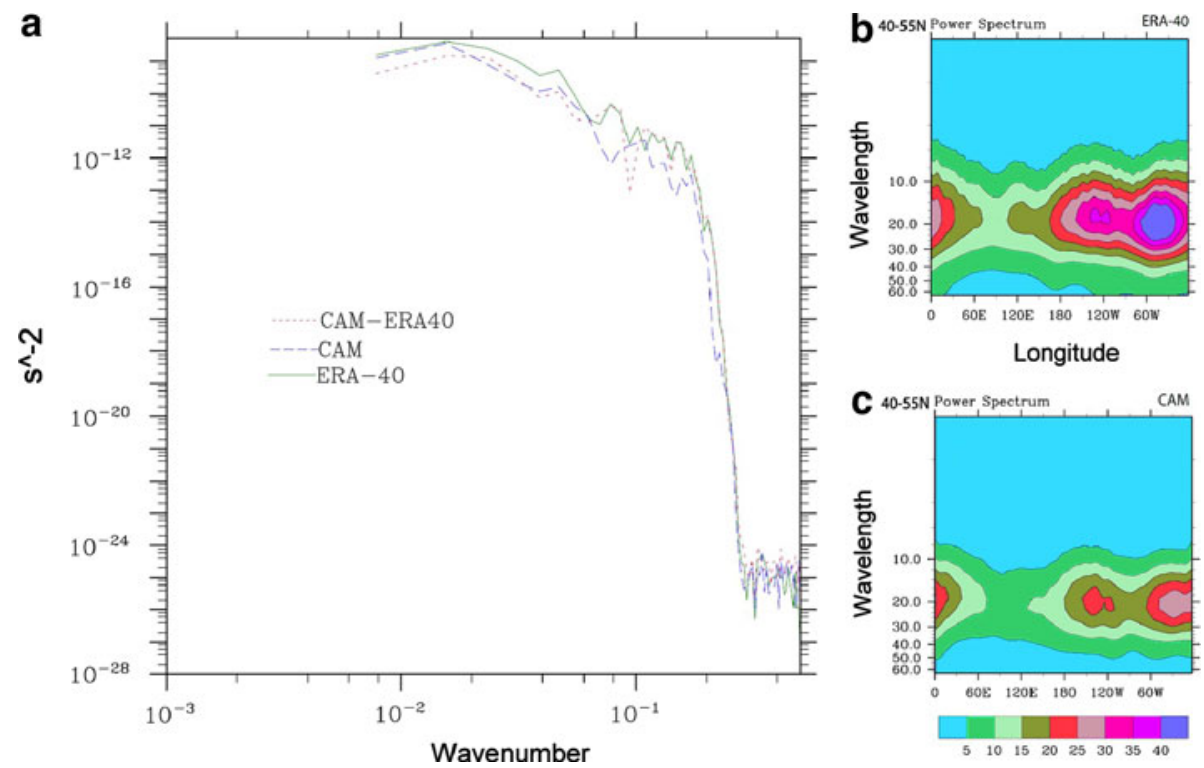

Fig. 2 a Power spectra of vorticity at $40 \mathrm{~N}$ and $\sigma=0.3$ for ERA-40 (green line), CAM3 (blue dashed line), and the bias (red dotted line). For many wavenumbers CAM3 has smaller amplitude. Wavelet transform of high pass (2-8 days) vorticity in (b) ERA-40 and (c) CAM3 data averaged over the latitudes $40-55 \mathrm{~N}$. For (a) the horizontal axis is a wavenumber per 128 grid points, hence wavenumber $1=7.8 \times 10^{-3}$ while the vertical axis has units of

slight increase in scale (from wavenumber 8 to 7) from the upstream to downstream end of the NPST in ERA-40 data (Fig. 2b) but scale change is not obvious in the CAM3 (Fig. 2c) data. Neither ERA-40 nor CAM3 show noticeable scale shift for the NAST. The wavelet transforms (using the derivative of the Gaussian) might not be ideal indicators since along an individual latitude circle the trend varies. For example, along $40 \mathrm{~N}$ the length scale diminishes in ERA-40 from wavenumber 6 to 8 over the NPST. Along other latitudes the scale has no apparent change or increases. ERA40 and CAM3 differ most for the downstream end of the NAST when only a single latitude circle is used because of the large latitudinal error of the storm track.

Regarding the NAST, PGT find the proxy measure of $\overline{v^{\prime} T^{\prime}}$ to be narrower and in particular not extending as far north in CAM3 compared to ERA-40. PGT also find the proxy measure of $\overline{v^{\prime} T^{\prime}}$ to be more zonally-oriented in CAM3 and to extend further into western Europe, whereas this measure in ERA-40 extends northward over Iceland. The proxy measures used here: Ens' and $\mathrm{KE}^{\prime}$ have similar bias in location as $\overline{v^{\prime} T^{\prime}}$. Though the smaller magnitudes in CAM3 make it harder to see, both Ens' and $\mathrm{KE}^{\prime}$ appear to be narrower in CAM3. As mentioned, $\mathrm{KE}^{\prime}$ and Ens' tend to emphasize the downstream end of the storm track and biases shown in Fig. 1 clearly show these proxy measures extending much further east and south over southern Europe and the Mediterranean Sea in CAM3. (This eastward vorticity variance, $\mathrm{s}^{-2}$. The vertical axis is also normalized so that the area under the curve equals the variance of the detrended series. For (b) and (c) the horizontal axis is longitude while the vertical axis is a length scale in terms of grid intervals for the 128 grid points along a latitude circle. Hence, 10.0 means a size of 10 grid intervals. The contour values of the color bar are in units of $10^{-10} \mathrm{~s}^{-2}$

extension of the storm track is less evident in $\overline{v^{\prime} T^{\prime}}$ shown by PGT since that proxy measure emphasizes the early stages of frontal cyclones.) The distance separating the tracks grows as one looks further downstream. Near the east coast of North America, the distance between ERA-40 and CAM3 tracks is a couple of degrees latitude. Where the tracks cross the Greenwich meridian, the difference grows to about 10 degrees latitude. The Ens' proxy fields have slightly larger separation between the CAM3 and ERA-40 storm tracks than do the $\mathrm{KE}^{\prime}$ proxy fields. Both the $\mathrm{KE}^{\prime}$ and Ens' fields of CAM3 have a secondary maximum in southern Europe and the Mediterranean Sea that is not present in ERA-40 data. Consequently, the CAM3 storm track seems to be longer as well as much further south on the downstream end of the track. At 30E, the CAM3 track is about 15 degrees south of the ERA-40 maximum in both $\mathrm{KE}^{\prime}$ and $\mathrm{Ens}^{\prime}$. $\mathrm{KE}^{\prime}$ along the storm track is much less in CAM3, however the track separation and secondary maximum are large enough to cause a positive $\mathrm{KE}^{\prime}$ bias across the Mediterranean Sea.

Regarding the NPST, Fig. 1 shows the track to have a similar curving path but it is 3-5 degrees further north in CAM3 in both $\mathrm{KE}^{\prime}$ and Ens'. Hence CAM3 has both proxy indicators of the track too far north across the Pacific and too far south across most of the Atlantic. Ens' values along the NPST are systematically about 3 times larger in ERA-40 than CAM3. The KE' pattern is a bit different from the Ens': 
$\mathrm{KE}^{\prime}$ has peak values in the mid Pacific in ERA-40 with secondary maximum at the North American west coast. CAM3 has similar dual maxima, but with opposite emphasis, CAM3 has larger values at the downstream end of the NPST. These results are consistent with the $v^{\prime} T$ storm track proxy results shown in PGT. PGT also show that the CAM3 surface heat fluxes are markedly smaller off the east coast of Asia perhaps reducing the intensification of frontal cyclones on the upstream end of the NPST.

The subtropical jet streams (Fig. 3) have bias near the NAST and NPST that is consistent with the storm tracks. The north Atlantic jet crosses the North American east coast at nearly the same location in CAM3 and ERA-40 but it is much stronger and extends further east in CAM3. Consequently, the bias in zonal wind exceeds $10 \mathrm{~m} / \mathrm{s}$ across most of the north Atlantic, especially near and over western Europe (Fig. 3a-c). Correspondingly, the meridional wind, $v$ is more northward over the North Atlantic and less southward over Europe in ERA-40 (Fig. 3d-f). Across the north Pacific the CAM3 zonal wind component is again stronger, but not by as much as over the Atlantic. Contrary to the north Atlantic, the stronger winds at the downstream end of the NPST (in the northeastern Pacific) are further north in CAM3. As for the meridional wind in the NPST, east of the dateline the pattern is opposite to the $\mathrm{v}$ bias found along the NAST, but the cause is largely due to a phase shift error. CAM3 has more northward motion shifted further west compared to ERA-40 data leading to the northeastern Pacific dipole pattern in $v$ bias (Fig. 3f).

$$
\text { a }
$$

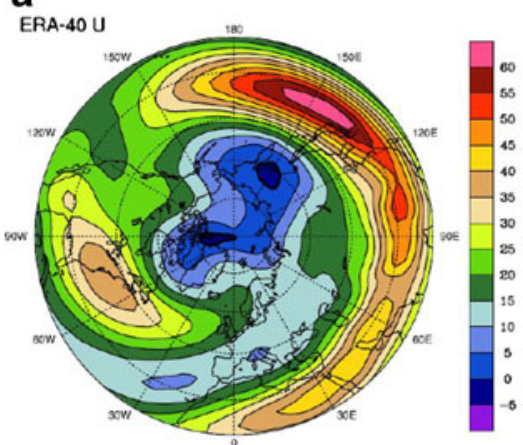

b

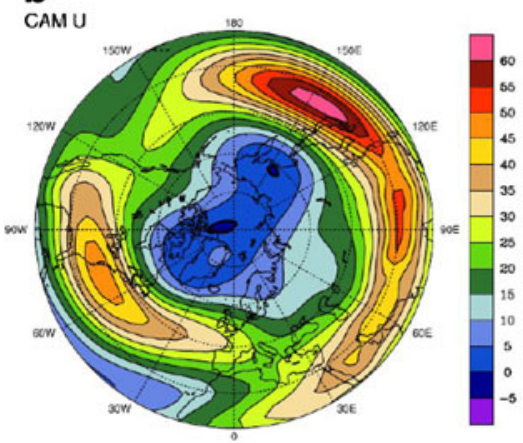

CAM-ERA40

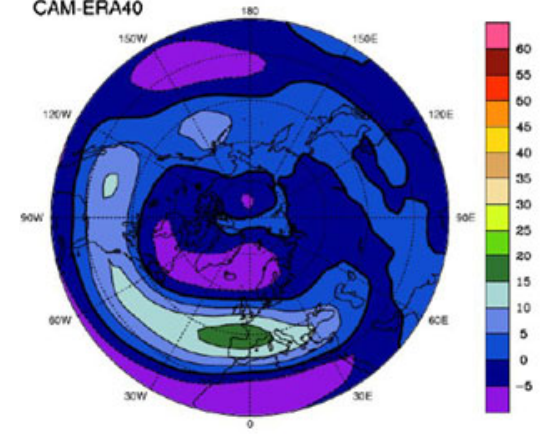

At sigma $=0.3$

d

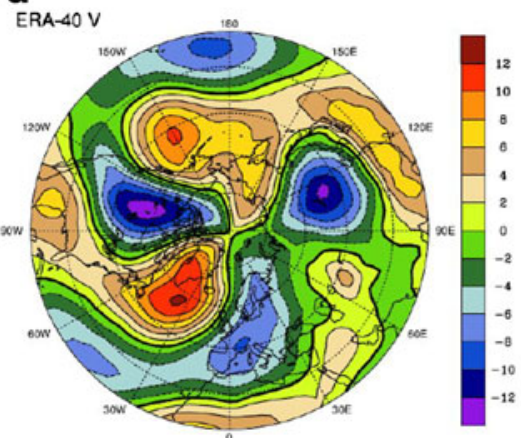

e

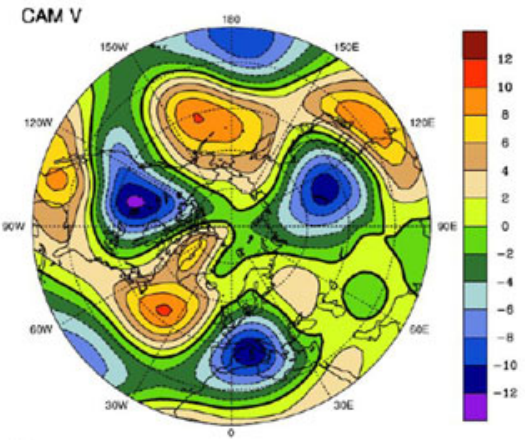

f

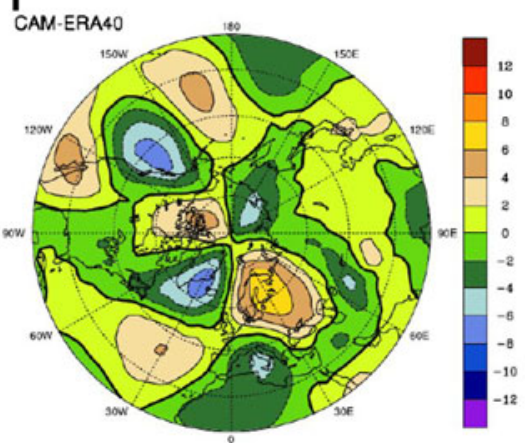

At sigma $=0.3$

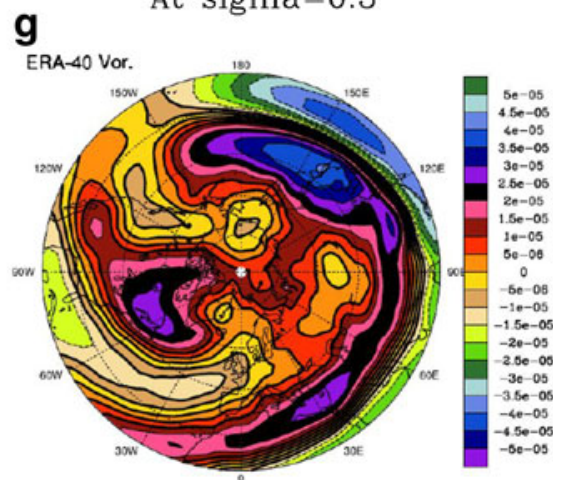

h

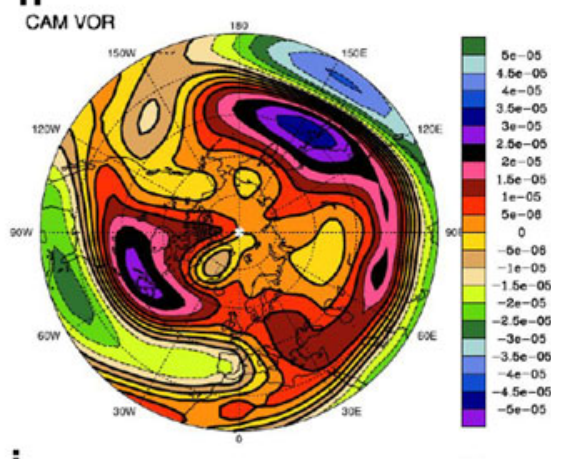

i

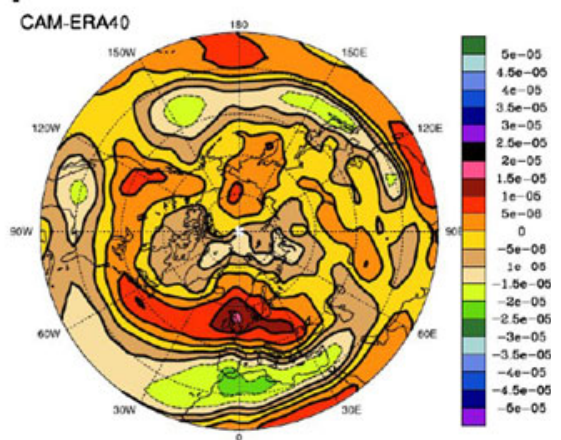

Fig. 3 Zonal (left column, a-c), meridional (middle column, d-f) components of the wind in $\mathrm{m} / \mathrm{s}$, and relative vorticity (right column, g-i) at level $\sigma=0.3$ during DJF in $\mathrm{s}^{-1}$. Top row: ERA-40 data, middle row: CAM3 20 year simulation, bottom row: bias 
Unlike the Pacific subtropical jet, CAM3 does noticeably less well simulating the north Atlantic jet stream. Dynamically, a stronger flow across the Atlantic might also lead CAM3 to move frontal systems too quickly across the north Atlantic causing: (a) the lows (vorticity maxima) to reach Europe more easily because (b) the intensification of the lows has had less time to amplify the ridge ahead of each trough (by warm advection). To the extent that meridional transient heat flux, $\overline{v^{\prime} T^{\prime}}$ is a measure of frontal cyclone baroclinic growth, PGT found smaller peak values of $\overline{v^{\prime} T^{\prime}}$ in the CAM3 data (by 20-25\%). Since PGT found $\overline{v^{\prime} T^{\prime}}$ to be elongated zonally, extending more into western Europe and less into the GIN Seas, then the heat flux bias is positive on the downstream end of the NAST and NPST. Those results are consistent with systems having lower $\mathrm{KE}^{\prime}$ and $\mathrm{Ens}^{\prime}$ in the CAM3 data.

Another point can be made about the smoothness of the patterns. Though all fields are regridded to the same resolution, the vorticity pattern in ERA-40 has proportionally larger amplitude in small scale waves than does CAM3. This can be seen in the smoother pattern of CAM3 vorticity (Fig. 3h) compared with ERA-40 data (Fig. 3g). ERA-40 was generated using a model with T63 resolution compared to T42 of the CAM3 simulations used here. However, it is clear from the vorticity fields (Fig. 3g-i) that while the larger scale pattern is similar between model and reanalysis, the amplitude is generally less in the CAM3 data, even for those large scale parts of the pattern.

\section{Vorticity equation terms and bias}

The bias of individual terms of the vorticity Eq. 1 are discussed first and provide insight into each group of terms in the vorticity bias equation.

\subsection{Ranking of individual terms in Eq. 1}

It is useful to begin the discussion with the general sizes and distribution of the vorticity equation terms. One might write the time mean of Eq. 1 in scalar form:

$$
\begin{aligned}
u \frac{\partial \zeta}{\partial x}+v \frac{\partial \zeta}{\partial y}+\beta v+\omega \frac{\partial \zeta}{\partial p}= & \underset{r}{\zeta p}+f \frac{\partial \omega}{\partial p}-\frac{\partial \omega \partial v}{\partial x \partial p}+\frac{\partial \omega \partial u}{\partial y \partial p} \\
& +F \zeta
\end{aligned}
$$

where ' $x$ ' refers to the zonal and ' $y$ ' to the meridional independent variable in spherical coordinates and derivatives are those relevant for spherical coordinates. Relative size varies geographically, but away from high topographic features, the terms are generally largest in the midlatitude oceanic storm tracks. Large amplitude topographic features often create dipolar patterns in those terms of Eq. 5 that involve $\omega$. Since topography varies between the CAM3 and ERA-40 models and $\sigma$ surfaces have large slopes near large topographic features, values of terms including $\omega$ and especially the bias should not be emphasized from those regions. Hence large dipolar values straddling high topographic features (e.g. Greenland) are not considered when making these rankings. One might rank the terms based upon the largest peak amplitude, but the peak values can be isolated and such a ranking might mask the overall contribution from each term. Instead, our ranking is based on an area-weighted average of the absolute value of each term for near sea level $(<10 \mathrm{~m}$ elevation) grid points within the domain from longitude $150 \mathrm{E}$ eastward to the Greenwich meridian and from 30 to $60 \mathrm{~N}$ latitude.

Each term in Eq. 5 is ranked by size as indicated in Table 1. The ranks differ from upper and lower troposphere so two representative levels are shown. A smaller rank means a larger magnitude term. Ranking varies with level and between ERA-40, CAM3 and the difference (bias). Table 1 samples the upper troposphere $(\sigma=0.3)$ near tropopause level where vertical motion tends to be small compared to lower tropospheric levels. The lower troposphere represented in Table 1 by the $\sigma=0.7$ columns, near where vertical motion has maximum amplitude.

Generally, most of the terms in Eq. 5 have larger values along the middle and downstream ends of the NAST and NPST. These are locations where the subtropical jet streams have entrance, peak value, and exit regions. Hence jet streak dynamics will be seen to cause a large portion of the larger amplitude (and some cancellation between) some of the terms. These are also locations where individual extra-tropical lows tend to have larger amplitude. Hence, results in Grotjahn (1996) are also relevant; he evaluated vorticity equation terms and composited the results from instantaneous data for 15 mature but still developing lows in the north Pacific. Grotjahn (1996) found the horizontal advection terms to be largest in the upper troposphere and the divergence term to be second largest in the upper troposphere and the largest term at low levels. Secondary in magnitude are vertical advection terms (especially notable around the $700 \mathrm{hPa}$ level) and tilting terms (but significant tilting terms values have small areal extent and there would be some cancelling between positive and negative areas as storms move). In short, Grotjahn (1996) finds similar variation with height as is seen in the ranking for the time average data used here.

In the upper troposphere, the largest values are reached by the three parts of the horizontal advection of absolute vorticity and the quasi-geostrophic divergence term; the other terms tend to be of secondary importance. These rankings hold for ERA-40 and CAM3 data. (However, the relative vorticity part of the divergence term has a bit more prominence in the CAM3 data.) These rankings are not too 
surprising since most of the higher ranked terms are just those present in the quasi-geostrophic system. These rankings give a sense of the relative peak values reached over the Northern Hemisphere, but the ranking of the largest terms change somewhat between different regions. Also, while the two horizontal advection terms are individually largest, much cancellation occurs between these two terms as explained below. The bias at upper levels has a similar ranking as the individual terms.

In the lower troposphere the rankings differ somewhat from the upper troposphere and differ more strongly between ERA-40 and CAM3. At this level, the vertical advection and advection of planetary vorticity terms are largest (in ERA-40) followed by the quasi-geostrophic divergence and horizontal advection of relative vorticity terms. CAM3 has a partly different ranking, advection of planetary vorticity and quasi-geostrophic divergence terms are highest-ranked followed by the vertical advection of vorticity term and a tilting term. The two tilting terms have some large peak values along the two storm tracks, however, there is much cancellation between them. The bias has some tendency to be larger where the rankings differ between ERA-40 and CAM3. However, the bias is largest for the zonal advection, quasi-geostrophic divergence, advection of planetary vorticity, and meridional advection terms.

An important result in Table 1 is that the friction residual term, ranks low in both models and in the bias at both levels. If the friction residual had been prominent, then a notable fraction of the vorticity balance might have been contributed for by unaccounted for processes. However, the highest ranking of the friction residual term is 5th for the bias. The low ranking of the friction residual holds even when averages over an Arctic region (near sea level grid points poleward of $70 \mathrm{~N}$ ) are included for consideration.

\subsection{Upper tropospheric patterns and bias}

The larger values of $v \partial \zeta / \partial y$ (rank 1 in Eq. 5) are positive and occur over southern North America and over northern Africa (Fig. 4a, b). The larger values of $u \partial \zeta / \partial x$ (Fig. 4d, e) have similar distribution but opposite sign at upper levels. The $\sigma=0.3$ level is shown; the pattern at $\sigma=0.5$ is very similar (but half the amplitude). These primary maxima are on the upstream end of the two subtropical jet streams. The jet stream in each place has a west-southwest to eastnortheast orientation. Coupled with relative vorticity mainly due to shear, the two components of the horizontal advection of relative vorticity are individually large both places. (Specifically: $u>0$ with $\partial \zeta / \partial x<0$ and $v>0$ with $\partial \zeta / \partial y>0$; see relevant parts of Fig. 3.) The flow is largely perpendicular to the vorticity gradient hence the two terms largely cancel and the cancellation suggests the bias flow is largely geostrophic. One can see less northward advection in the Atlantic adjacent to northern Europe (between 0 and $30^{\circ} \mathrm{W}$ ) in CAM3 (Fig. 4a, b, d, e). Due to a southward shift in CAM3, the zonal advection has a dipolar pattern over Eastern Europe and Mediterranean with positive bias south of negative bias.

Horizontal advection of relative vorticity using CAM3 data is generally similar to the ERA-40 results (but about 10-20\% less magnitude due to the smaller magnitude in CAM3) with some shifting of the positions of largest values. The primary exception is that the zonal advection near the East Asian subtropical jet has stronger small scale fluctuation in ERA-40 (Fig. 4d). As for the position shift, CAM3 data have peak values of both terms that are up to 5 degrees latitude further north and slightly downstream from the ERA-40 locations over southern North America, across northern Africa, and across the north Pacific. (The meridional and zonal advections still largely cancel each place). However, positive meridional advection north of
Table 1 Vorticity equation terms ranking DJF, northern hemisphere middle latitudes

Areas of steep or high topography, such as near Greenland excepted Values in parentheses are area-weighted averages in $\mathrm{NH}$ storm track regions $(30-60 \mathrm{~N}, 150 \mathrm{E}-$ 360 E) over ocean. The unit is $10^{-11} \mathrm{~s}^{-2}$

a Ties mean that average values differ by 0.2 or less and thus are essentially the same

\begin{tabular}{|c|c|c|c|c|c|c|}
\hline \multirow{2}{*}{$\begin{array}{l}\text { Level } \\
\text { Data }\end{array}$} & \multicolumn{3}{|l|}{$\sigma=0.3$} & \multicolumn{3}{|l|}{$\sigma=0.7$} \\
\hline & ERA-40 & CAM3 & Bias & ERA-40 & CAM3 & Bias \\
\hline$v \frac{\partial \zeta}{\partial y}$ & $1^{\mathrm{a}}(11.37)$ & $1(11.61)$ & $1^{\mathrm{a}}(11.12)$ & $4(4.38)$ & $6(2.62)$ & $4(2.81)$ \\
\hline$u \frac{\partial \zeta}{\partial x}$ & $1^{\mathrm{a}}(11.35)$ & $2(7.65)$ & $1^{\mathrm{a}}(11.18)$ & $5(3.65)$ & $5(3.00)$ & $1^{\mathrm{a}}(3.62)$ \\
\hline$f \frac{\partial \omega}{\partial p}$ & $3^{\mathrm{a}}(7.99)$ & $3(5.87)$ & $4(4.29)$ & $3(4.88)$ & $1^{\mathrm{a}}(3.86)$ & $1^{\mathrm{a}}(3.63)$ \\
\hline$\beta \mathrm{v}$ & $3^{\mathrm{a}}(7.81)$ & $4^{\mathrm{a}}(4.72)$ & $3(6.18)$ & $1^{\mathrm{a}}(5.49)$ & $1^{\mathrm{a}}(3.87)$ & $3(3.31)$ \\
\hline$\zeta \frac{\partial \omega}{\partial p}$ & $5(3.56)$ & $4^{\mathrm{a}}(4.90)$ & $7^{\mathrm{a}}(1.46)$ & $8^{\mathrm{a}}(0.88)$ & $9(0.19)$ & $8^{\mathrm{a}}(0.73)$ \\
\hline$\omega \frac{\partial \zeta}{\partial p}$ & $7^{\mathrm{a}}(1.08)$ & $6^{\mathrm{a}}(2.50)$ & $7^{\mathrm{a}}(1.46)$ & $1^{\mathrm{a}}(5.56)$ & $3^{\mathrm{a}}(3.59)$ & $6(2.00)$ \\
\hline$\frac{\partial \omega \partial v}{\partial x \partial p}$ & $7^{\mathrm{a}}(0.92)$ & $6^{\mathrm{a}}(2.50)$ & $5^{\mathrm{a}}(1.58)$ & $6(2.78)$ & $3^{\mathrm{a}}(3.68)$ & $8^{a}(0.93)$ \\
\hline$\frac{\partial \omega \partial u}{\partial y \partial p}$ & $9(0.32)$ & $9(1.02)$ & $9(0.80)$ & $8^{\mathrm{a}}(1.05)$ & 7 (2.29) & 7 (1.26) \\
\hline Fr & $6(1.84)$ & $8(1.59)$ & $5^{\mathrm{a}}(1.69)$ & $7(2.34)$ & $8(1.36)$ & $5(2.51)$ \\
\hline
\end{tabular}




\section{a $\quad \sigma=0.3$}

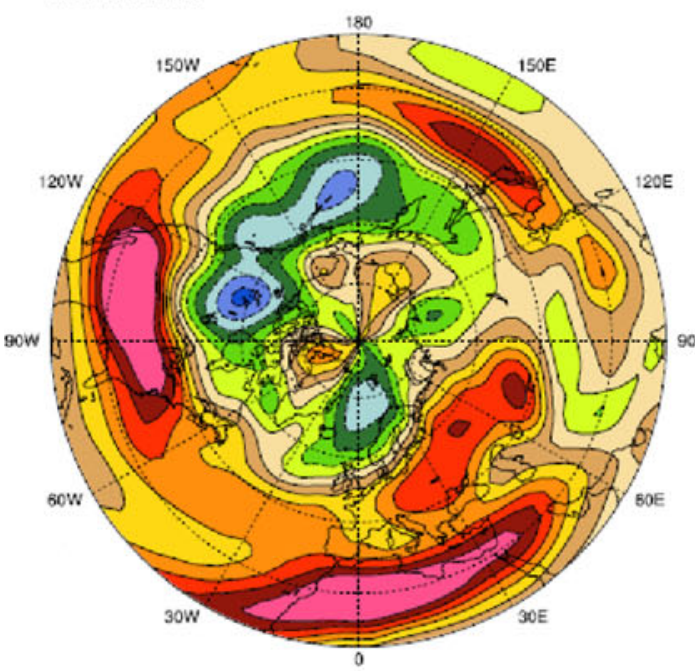

b

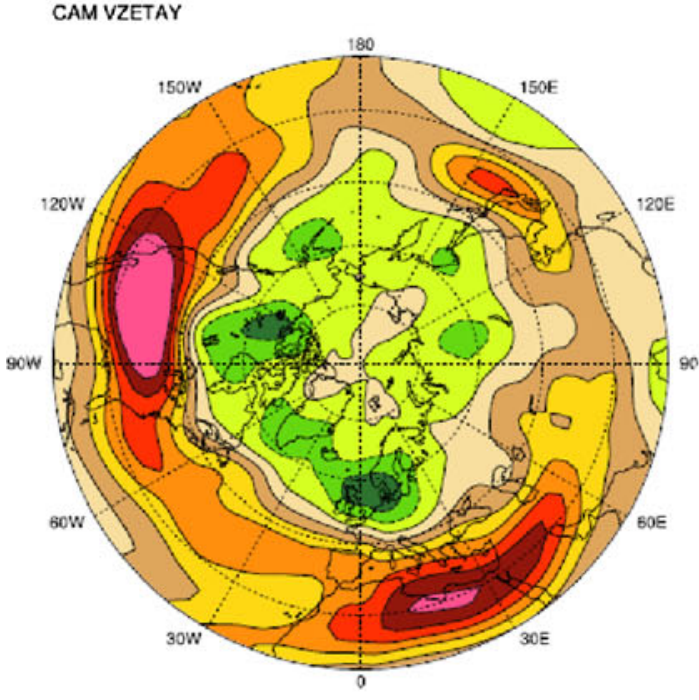

C

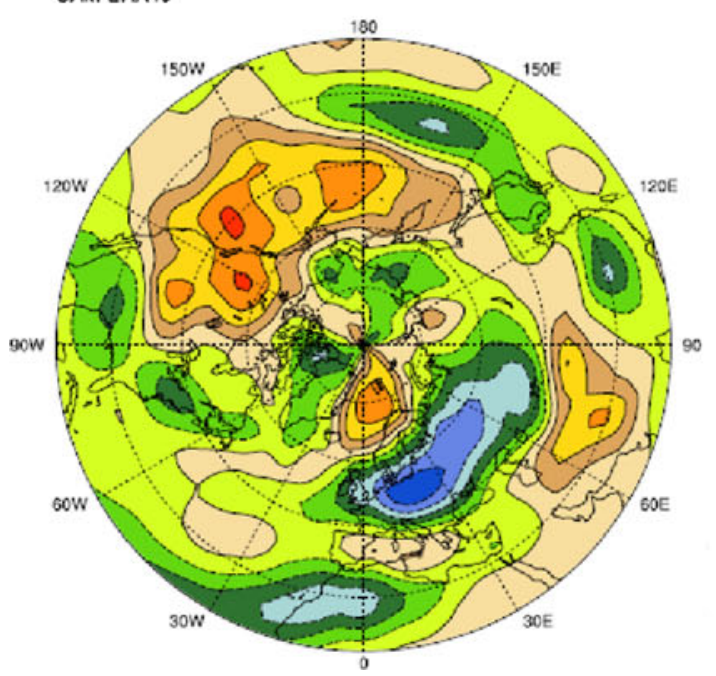

$\mathrm{d}_{\mathrm{ERA}-40 \mathrm{ZZETAX}} \sigma=0.3$

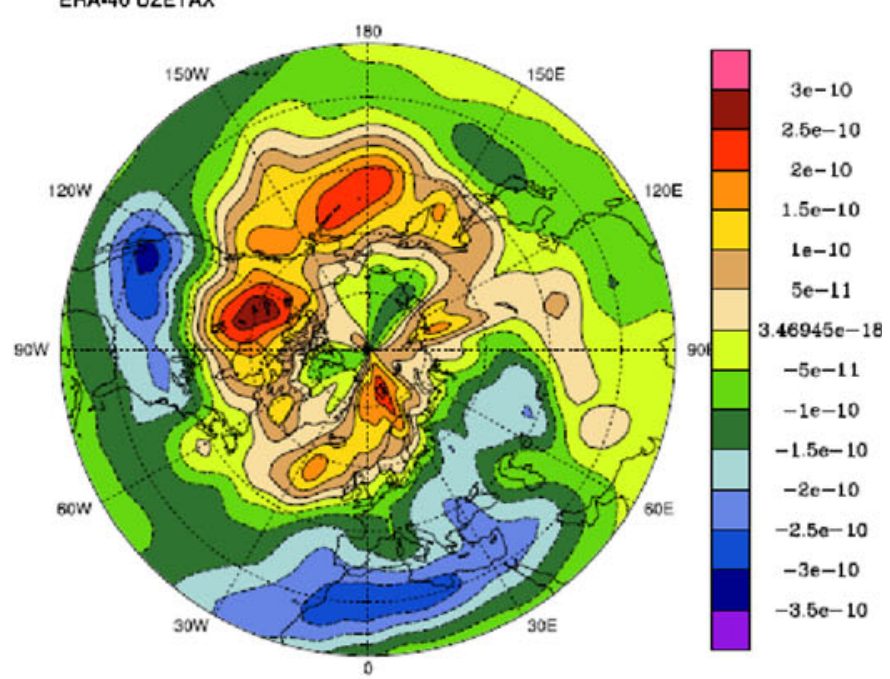

CAM UZETAX

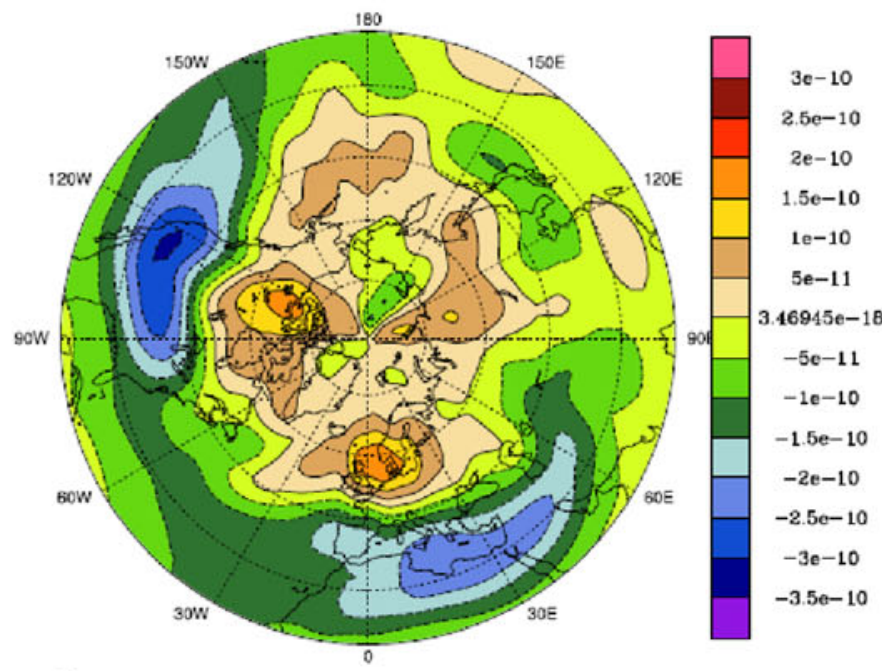

f

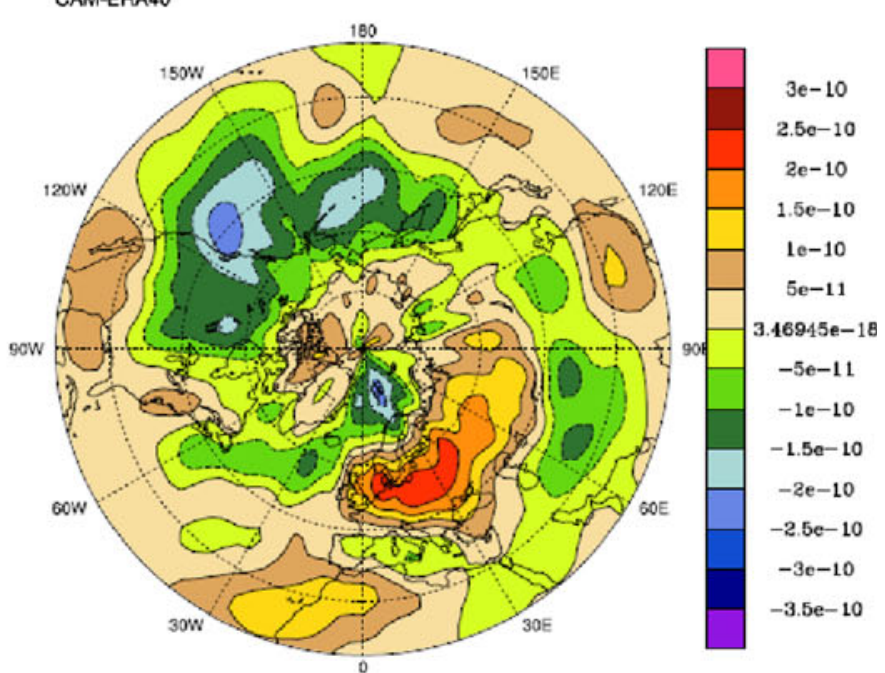


4 Fig. 4 Comparison of the two horizontal components of relative vorticity advection (a-f) in the vorticity equation. CAM3 data, ERA40 data, and the corresponding bias (CAM3-ERA-40). The level is $\sigma=0.3$ and the units are $\mathrm{s}^{-2}$. $\mathbf{g - i}$ Quasi-geostrophic divergence term and $\mathbf{j}-\mathbf{l}$ Friction residual at $\sigma=0.3$ Notice how this residual term, presumably mainly due to friction is so much larger in CAM3 than ERA-40 over east Asia, the central Pacific, and North Sea

Europe is centered more than 20 degrees further south in CAM3; again there is cancellation between meridional and zonal advection, but perhaps not quite as much as elsewhere. Near the east coast of Asia, the small scale variation in ERA-40 data (not found in CAM3) appears in the meridional advection bias.

Peak values for the bias of the individual horizontal advection terms are about half peak values of each term for either dataset (CAM3 or ERA-40). This sizable bias is related to qualitatively small biases in the geopotential height field (not shown). Over Europe and adjacent regions the dipolar pattern seen in the bias (Fig. 4c, f) results from the stronger flow being narrower in latitude across that region in CAM3. The reanalysis data have a more diffluent flow with a hint of a trough near the North African Atlantic coast that is not present in CAM3 and with more northward motion over the North Sea than in CAM3. A corresponding result is a $300 \mathrm{hPa}$ geopotential height bias (not shown) of a trough over the North Sea and a ridge over North Africa. That height bias affects both the horizontal wind and vorticity gradient over the region. Those height bias properties of stronger zonal flow and less northward flow on the north side of the jet stream are consistent with the CAM3 model tendency to have the NAST narrower (in latitude) and further south into Europe than in the reanalysis data. In other words, the geostrophic flow bias from a narrower jet near the west coast of Europe results in a negative (positive) geopotential height bias over Europe (Northern Africa), with a corresponding positive (negative) vorticity bias as shown in Fig. 3i. This dipole vorticity bias also results in the dipole pattern in the advective tendency evident in Fig. 4.

The sum of the two horizontal advections (Fig. 4a plus d; Fig. 4b plus e) largely favors the meridional advection for latitudes from 20 to $45 \mathrm{~N}$. Poleward of that the two terms more nearly cancel. The horizontal advection bias (Fig. 4c plus f) looks more like the meridional advection bias over the NPST, North America, and Europe (including the North Sea) and more like the zonal advection bias over the north Atlantic.

From a simple omega equation analysis (e.g. Carlson 1991, Sect. 14.1) a straight jet entrance region will tend to have vertical motion below: rising on the right side and sinking on the left side (viewed looking downwind). Near the tropopause, that vertical motion requires divergence on the right entrance and convergence on the left entrance regions. The quasi-geostrophic divergence term, $f \partial \omega / \partial p$ will be positive on the left entrance and negative on the right entrance; both ERA-40 and CAM3 have that pattern over East Asia and adjacent Pacific. Over North America, this pattern is prominent for CAM3 (Fig. 4h) but it is less obvious for ERA-40 (Fig. 4g). The north Atlantic jet stream is much stronger in CAM3 so much of the pattern associated with acceleration of the subtropical jet in that region reappears in the bias (Fig. 4i). On the downstream end of the NAST the negative divergence term over Europe in CAM3 is again 10 degrees or more south of its location in ERA-40 data, consistent with the jet stream and horizontal advection biases. The north Pacific jet stream has much less bias, but the quasi-geostrophic divergence term again has a bias similar to the CAM3 pattern (but smaller amplitude) in the western north Pacific, but not further downstream.

\subsection{Lower tropospheric patterns and bias}

Table 1 indicates the relative sizes of the vorticity equation terms at the representative lower tropospheric level, $\sigma=0.7$. The individual rankings of the terms in both ERA-40, CAM3, and the bias differ, but most of the same terms are ranked in the top 5. Figure 5 plots the five largest terms at $\sigma=0.7$ based on their size in the ERA-40 data plus the friction residual. The discussion that follows considers those terms.

Figure 5a-c shows the vertical advection of relative vorticity in ERA-40, CAM3 and the bias. The reanalysis and model data have distinct, elongated, negative regions that align well with the NPST and NAST. Following the discussion in Grotjahn (1996) the sign of the term is often linked to whether tilt or amplitude change with height dominates the vertical derivative of vorticity. Since the pattern is strongly negative along the storm tracks, tilt of the vorticity axes, upstream with height, is the dominant effect at this level. The CAM3 model has much weaker time mean vertical advection, about half the ERA-40 values. Hence the bias is strongly positive along both the NPST and NAST.

Figure $5 \mathrm{~d}-\mathrm{f}$ shows the meridional advection of planetary vorticity, or $v \beta$ term in ERA-40, CAM3 and the bias. This reanalysis and model data have distinct dipoles consistent with poleward and equatorward flow on either side of troughs in the planetary wave pattern (wavenumber 3 being prominent). Hence the mid and upper level troughs over the eastern sides of the continents have northerly motion over the continent $(v \beta<0)$ and the opposite over the adjacent ocean. The magnitudes of the extrema in CAM3 are similar to those in ERA-40 so the bias is relatively small compared with other vorticity equation terms. 


\section{g $\quad \sigma=0.3$ \\ ERA-40 FOMEGAP}

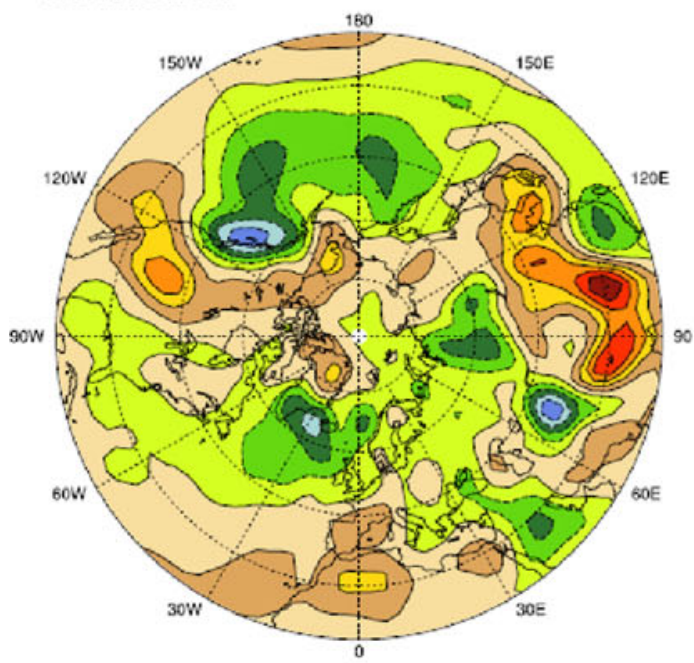

h

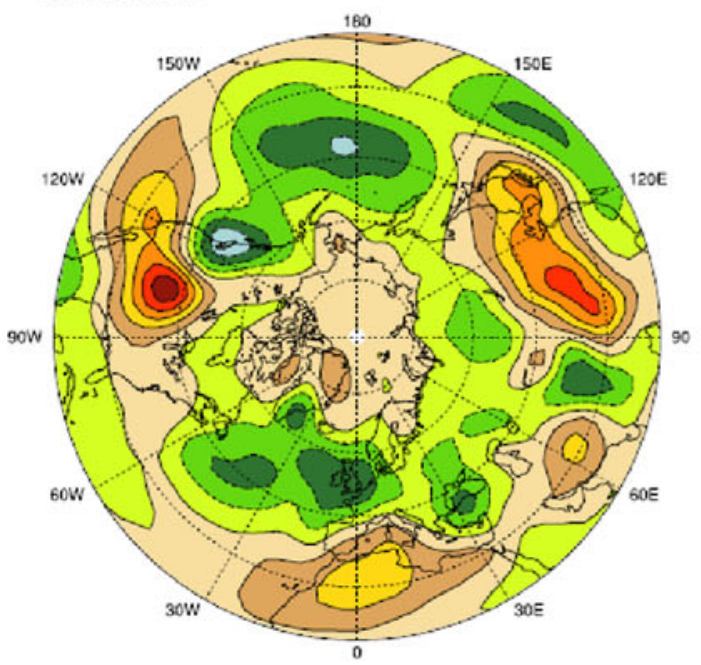

i

CAM-ERA40

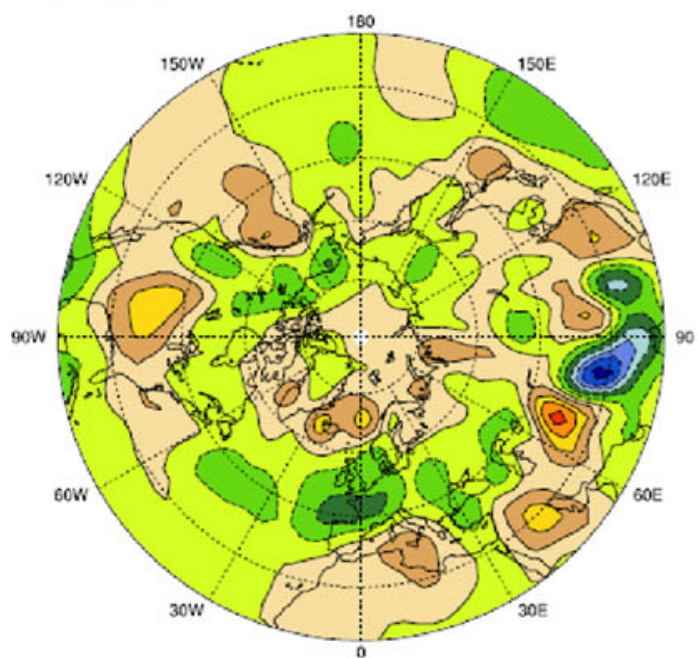

j $\quad \sigma=0.3$

ERA-40 $\mathrm{Ft}$

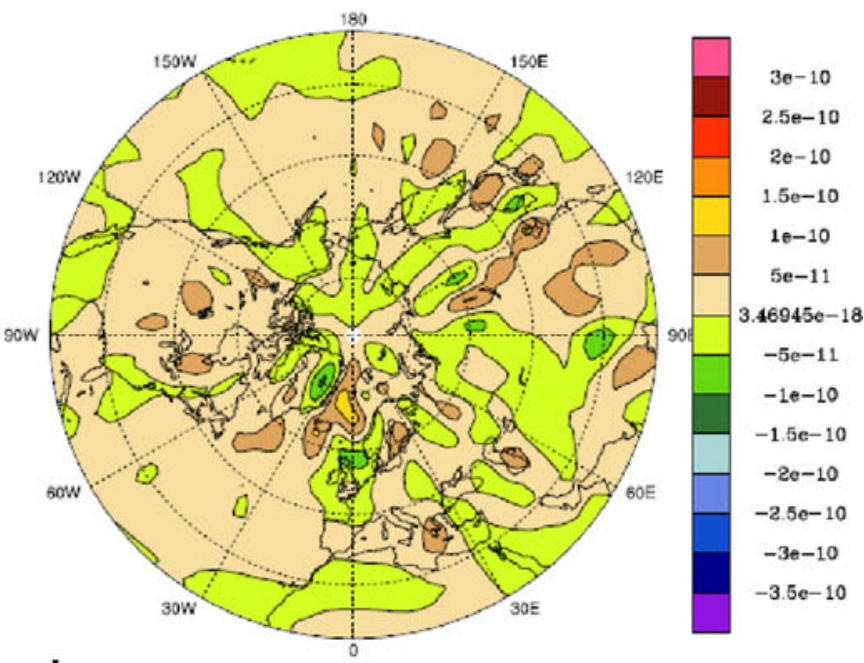

k

CAM Fr

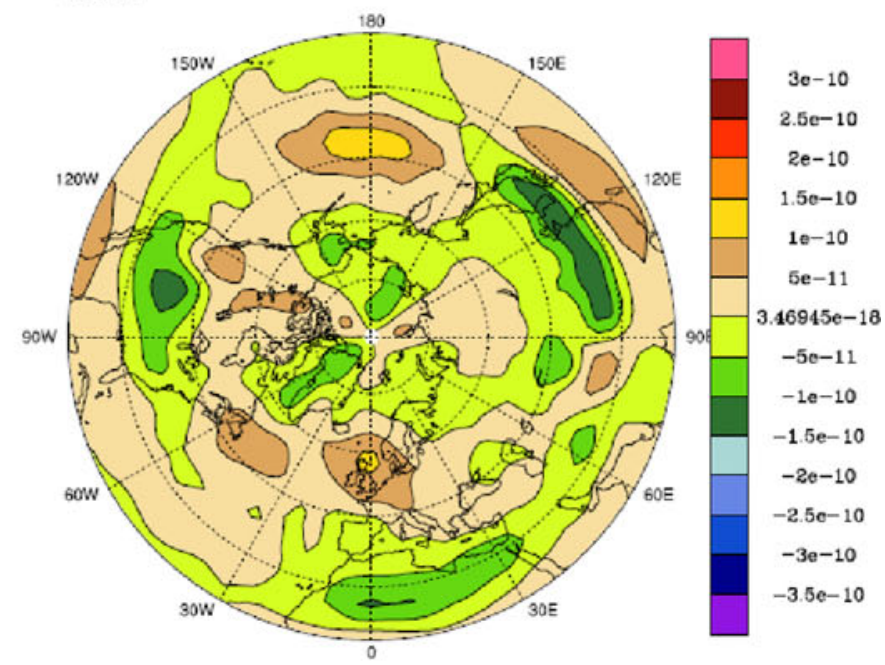

I

Frlctlon(ressdual)

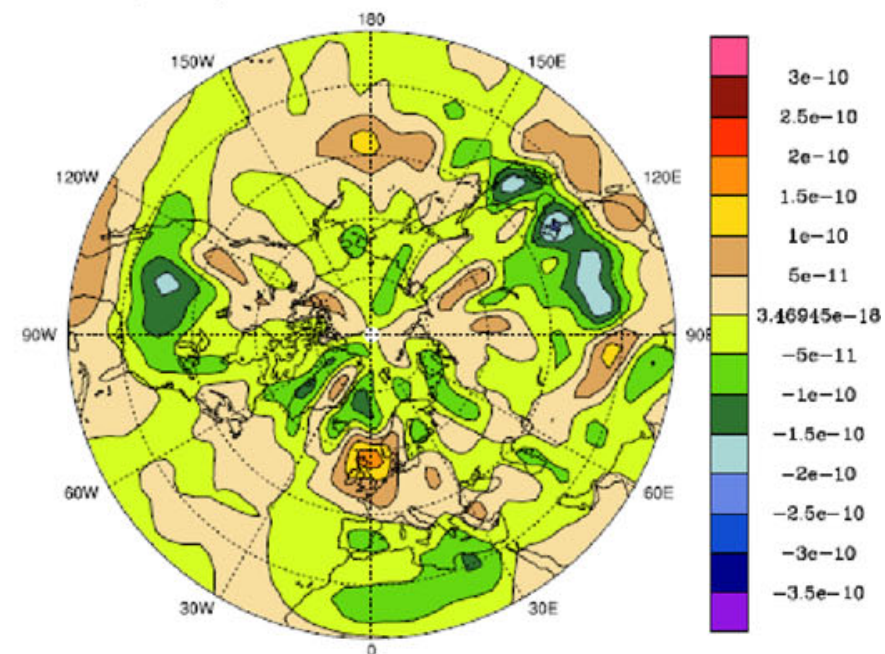

Fig. 4 continued 
There are a few differences of note. The ridge centered near the west coast of North America is narrower in ERA40 so the dipole has narrower longitudinal range in ERA40. The northward flow over the North Atlantic is further north in ERA-40 and the southward flow downstream is weaker in ERA-40 than in CAM3, consistent with the downstream track being shifted equatorward in CAM3, as mentioned above. The pattern for $\mathrm{v} \beta$ in the upper troposphere was not shown because it is very similar to the pattern shown in Fig. 5d-f; the main differences are the magnitudes are larger at $\sigma=0.3$ especially in the subtropics (so that weak subtropical extrema found at $\sigma=0.7$ are comparable to midlatitude extrema at $\sigma=0.3$ ).

Figure $5 \mathrm{~g}-\mathrm{i}$ shows the meridional advection of relative vorticity term. The pattern in the reanalysis and model data is largely aligned with the NPST and NAST. The values along the storm tracks are roughly half again larger in ERA-40 than in CAM3. Also, the elongated band of maximum values is further north in CAM3 than in ERA40. However, there is quite a bit of cancellation between the meridional and zonal advection of vorticity. Specifically, in the first halves of the NPST and the NAST, about $2 / 3$ to $3 / 4$ of the positive meridional advection is cancelled by negative zonal advection: Fig. $5 \mathrm{~g}$ versus $5 \mathrm{p}$ for ERA-40 and $5 \mathrm{~h}$ versus $5 \mathrm{q}$ for CAM 3 . The cancellation is proportionally much less in the western north Pacific in ERA-40, but CAM3 differs with nearly complete cancellation in that region. Elsewhere, CAM3 has an elongated positive band over the Mediterranean Sea across southern Asia, but that pattern is either not present in ERA-40 or shifted 10 degrees further south (and 30 degrees westward) in ERA40. The meridional and zonal advections do not have such obvious cancellation for the bias. The location error of maximum values mentioned above results in negative bias parallel to, but equatorward of both storm tracks. Similarly, the planetary wave error near the Greenwich meridian shows up as negative bias over northwestern Africa.

Figure $5 \mathrm{j}-1$ shows the distribution of the residual term, which should be frictional processes. (However, being a residual, this term includes various friction, mixing, and diffusion processes as well as unresolved processes in the numerical evaluation of the vorticity equation that differ between ERA-40 and CAM3 due to their different spectral truncations.) In ERA-40, Fig. 5j, this term is negative over most of the middle and high latitudes, especially the first half of the NPST and NAST. In CAM3 the opposite is generally true: much of the middle and high latitudes has positive value and in the North Pacific and North Atlantic (including parts of the two storm tracks) the values are notably positive. Given the opposite signs, the bias is larger than in the ERA-40 or CAM3 data for this term but overall, the residual bias is the fifth largest bias term.
The friction residual for the upper troposphere is shown in Fig. $4 \mathrm{j}-1$, and it differs a bit from the pattern at $\sigma=0.7$. In ERA-40 the values are small with a high wavenumber variation and no clear sign along the storm tracks (or indeed over much of the northern hemisphere). In CAM3 the friction residual has distinct negative values elongated over the jet entrance regions of North Africa, East Asia, and North America. Near the dateline, Newfoundland, and Greenwich meridian CAM3 has large positive values. Hence the bias at $\sigma=0.3$ (Fig. 4l) looks much like the CAM3 residual (Fig. 4k). Large values of friction or diffusion would not seem likely at this level except perhaps where wind shear is strong (near the jet streams). Since larger values in these figures are not closely located near the strongest shear, we speculate that large values of this residual may be arising from nonlinear interaction of waves that are resolved by ERA-40 but not by our CAM3 simulation but nonetheless feed back onto scales that CAM3 resolves.

Figure $5 \mathrm{~m}-\mathrm{O}$ shows the quasi-geostrophic divergence term. The patterns are similar in ERA-40 and CAM3: peak values tend to be along the storm tracks and especially on the downstream ends. Vertical velocity tends to reach maximum amplitude near this level so one would not expect the divergence term to be large here. Values are positive indicating a preference for divergence in the indicated regions. The planetary ridge wavelength difference mentioned for the $v \beta$ term appears here as well, with CAM3 reaching a positive maximum further west (near $165^{\circ} \mathrm{W}$ ) than in ERA-40 (near $150 \mathrm{~W}$ ). That difference shows up as a negative bias in the northeastern Pacific.

Figure $5 \mathrm{p}-\mathrm{r}$ shows the zonal advection of relative vorticity. Along the NAST and NPST the advection is negative. As mentioned, this term is largely cancelled by the meridional advection. The wind speed $(|\vec{V}|)$ and the vorticity gradient magnitude $(|\nabla \zeta|)$ are both large for subtropical jets that have a northward as well as eastward orientation and that causes each component of the horizontal advection to be large. However, the two corresponding vectors $(\vec{V}$ and $\nabla \zeta)$ are nearly orthogonal to each other and that causes the two components of horizontal vorticity advection to cancel. The cancellation is less on the downstream ends of the storm tracks. Where the zonal advection term is negative in ERA-40 over northern Europe, it is positive in CAM3. The bias in zonal advection has a less clear pattern than the bias for the meridional advection. A strong dipole over Europe is caused by the latitudinal error of the downstream end of the NAST mentioned several times above. The bias in this term is generally worse for the NAST than for the NPST and positive in both regions. 


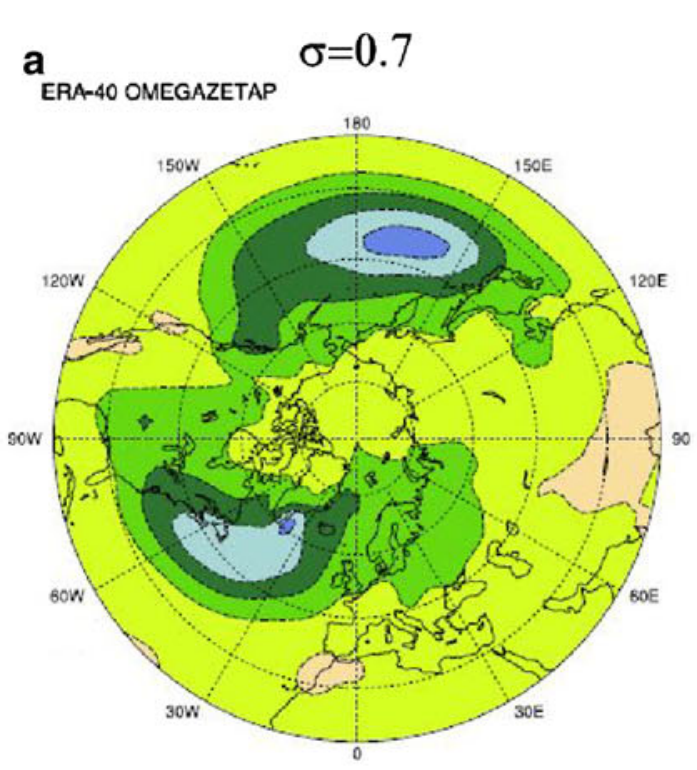

b

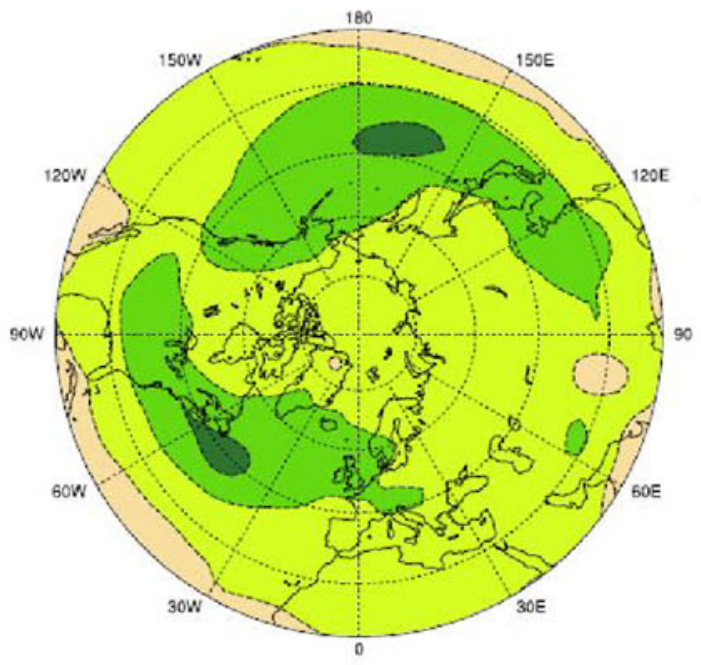

$\underset{\text { CAM-ERA4O }}{\text { C }}$

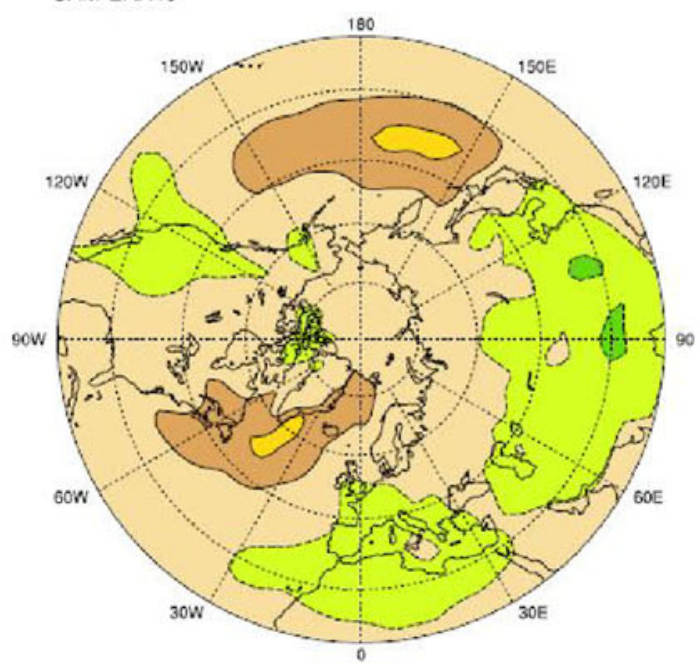

d $\quad \sigma=0.7$

ERA-40 VBETA

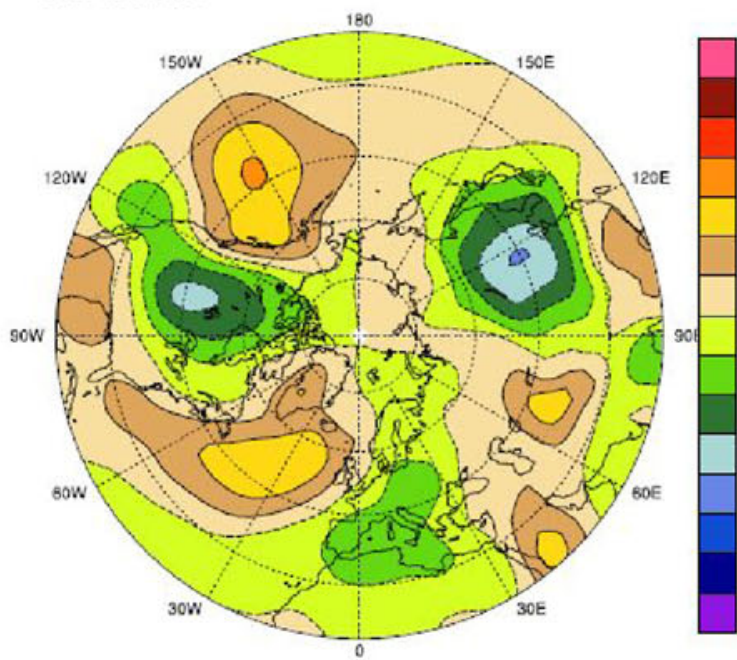

e

CAM VBETA

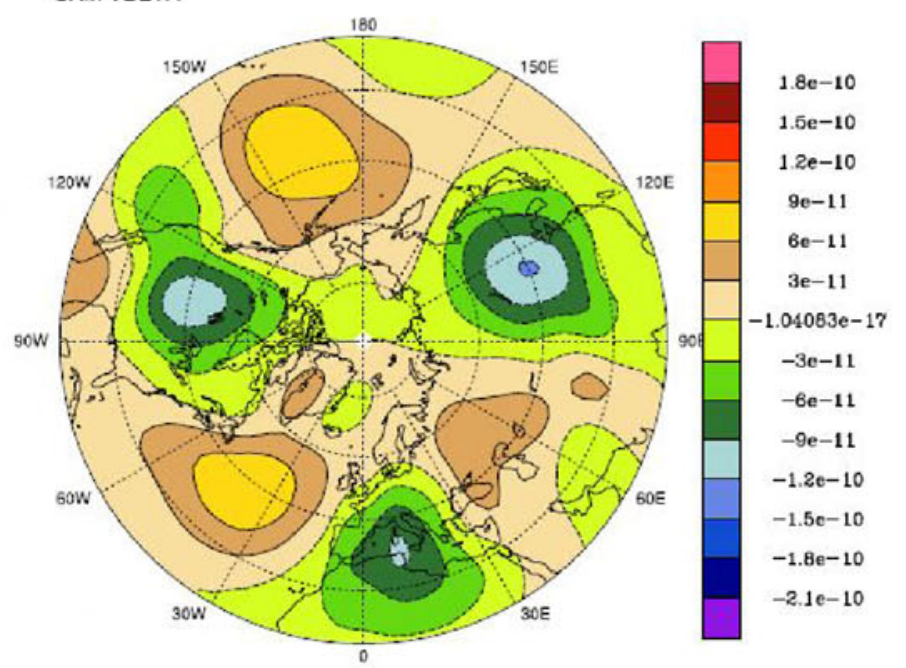

$f^{\prime}$

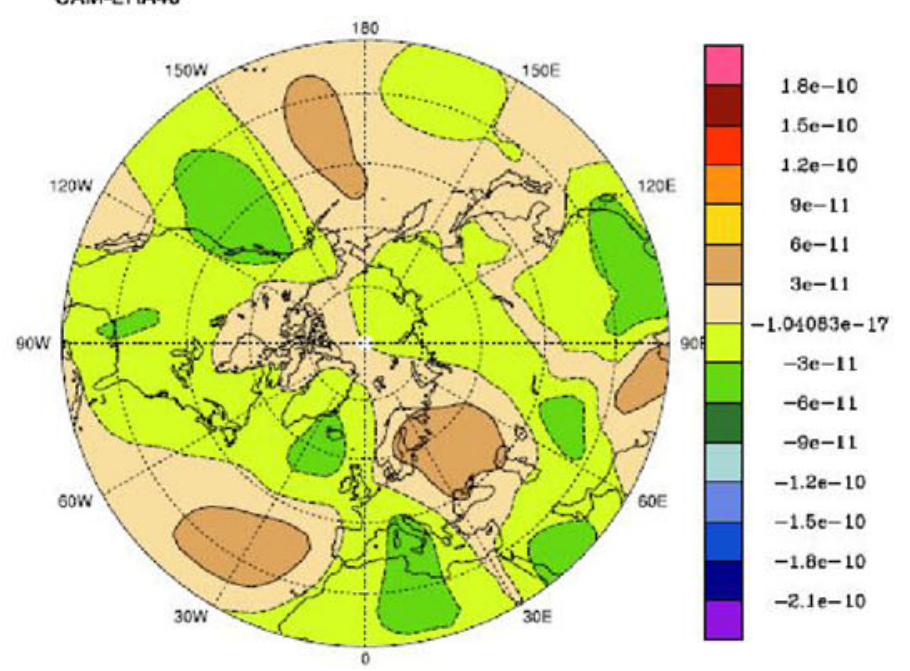


4 Fig. 5 Comparison of the larger terms in the vorticity equation between CAM3 and ERA-40 data, and the corresponding bias (CAM3-ERA-40). The level is $\sigma=0.7$ and the units are $\mathrm{s}^{-2}$. Panels (a-c) $\omega \partial \zeta / \partial p$; (d-f) $v \beta$; where these terms tend to be largest in ERA40 data. Panels $(\mathbf{g}-\mathbf{i}) v \partial \zeta / \partial y$; Panels $(\mathbf{j}-\mathbf{l})$ friction, calculated as a residual. Panels $(\mathbf{m}-\mathbf{o}) f \partial \omega / \partial p ;(\mathbf{p}-\mathbf{r}) u \partial \zeta / \partial x$

\section{Linear group, nonlinear group, and transient group forcing}

The terms in the vorticity bias equation 4 are partitioned into four different groups of terms: linear terms, nonlinear terms, transient heat flux terms contribution to the time mean, and a residual needed for balance that is presumably mainly indicative of friction and diffusion. Our approach in discussing these terms is twofold. First, we must assess the appropriateness of using a forced linear stationary wave model to understand the contributions to the bias; that model would solve the terms in the linear group subject to forcing by all the other terms. Results from using such a model to study the bias are beyond the scope of this article (since the model includes 3 other prognostic equations besides one for vorticity) but may be presented in a future article. Second, we can isolate physical processes that create portions of the bias by making this partitioning. In the previous section we discussed various contributions to different terms in the vorticity equation, but most of those terms have significant contributions by multiple phenomena. Bias may result from transient processes that contribute to the time mean, and for the vorticity equation these include vertical and horizontal vorticity fluxes and the relative part of the divergence term (the tilting terms have less contribution). The nonlinear terms arise if the bias strongly interacts with itself; if this collection of terms is large then the basis for using a linear model to study the bias becomes questionable. Also questionable would be our ability to make a linearization assumption that allows us to study parts of the bias. Since using the linear model equations is proposed for future study, we organize our discussion here to identify the contributors (from nonlinearity, transients, and residual term) to the linear terms.

The linear, nonlinear, and transient groups of terms are calculated from CAM3 and ERA-40 data. The signs of the terms are as indicated in Eq. 4. Therefore, the top plot in a column equals the sum of the other 3 members of that column. The friction and diffusion term is estimated by calculating the residual in the vorticity bias equation and is the same as presented in the previous section. The other terms are partitioned differently from the prior section. Before, all contributions to a bias plot for a specific term: linear in bias, nonlinear, transient parts were all lumped together. Here the terms are split and combined based upon whether they represent a linear operator upon the bias (the linear group) or not. Nonlinear refers to terms that are quadratic in the bias and transient terms have been band pass filtered.

The four groups of terms from Eq. 4 at $\sigma=0.3$ are shown in Fig. 6a-d. This level is chosen to represent the features of the upper troposphere and jet stream level. The level was also emphasized in PGT for the temperature bias equation allowing some comparison with our earlier paper. The representative lower troposphere level $(\sigma=0.7)$ is given by Fig. 6e-h. To facilitate comparison, the same contour interval is used in all panels. Again we ignore the response in the regions of large topographic features, such as Greenland, since topography varies between ERA-40 and CAM3 models. Equation 4 makes clear that the linear terms (top panel) should equal the sum of the other three 'forcing' terms (panels below the top panel). The bottom panel, labeled friction, is a residual and it has been shown for the lower level $(\sigma=0.7)$ in Fig. 5l. The linear terms have a complex pattern but some of its parts can be seen in the individual terms described in the previous section. The forcing is discussed first.

It is immediately clear that nonlinear bias terms are smaller than other term groupings at most places. PGT found the linear terms of the temperature bias equation to be the larger group and nonlinear terms to be proportionally small. The notable exception for the vorticity bias equation is the Iberian Peninsula; at $\sigma=0.3$ the nonlinear terms are about half the value of the linear terms, at $\sigma=0.7$ nonlinear terms exceed the linear terms there (and are balanced mainly by transient terms). The positive maximum over Iberia can be deduced from Fig. 3. The zonal wind bias is positive (Fig. 3c) while the vorticity bias zonal gradient is negative to the west of Iberia thus contributing to a positive nonlinear term there. The tilting term $\partial \omega / \partial y \quad \partial u / \partial p$ using bias winds also contributes to the positive nonlinear term there. It is apparent that the nonlinear contribution over Iberia is largely from the location error of the downstream end of the NAST.

The transient terms are larger along the NPST, and secondarily along the NAST; the sign reverses between upper and lower troposphere, being positive in the upper troposphere (Fig. 6c) and negative in the lower troposphere (Fig. 6g). The larger negative values at $\sigma=0.7$ over the NPST are partly canceled by positive values of friction (residual) leaving near zero values for the linear group near $45 \mathrm{~N}$ between 52 and $20 \mathrm{~W}$. Similarly, along the NAST at $\sigma=0.3$ negative transient terms are largely canceled by friction and nonlinear terms. A major contributor to the pattern at $\sigma=0.7$ is the vertical advection term (comparing Figs. $5 \mathrm{c}$ and $6 \mathrm{~g}$, and noting the sign change in Eq. 4). In the upper troposphere from 0 to $30 \mathrm{E}$ and poleward of $40 \mathrm{~N}$, the pattern in Fig. $6 \mathrm{c}$ is largely produced by the meridional advection of vorticity (Fig. 4c). 


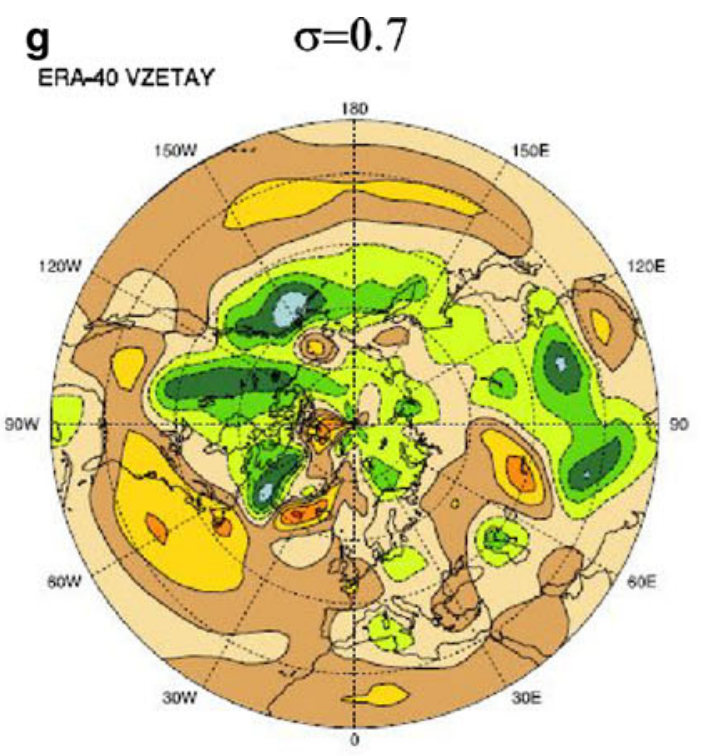

\section{h $_{\text {CAM VZETAY }}$}

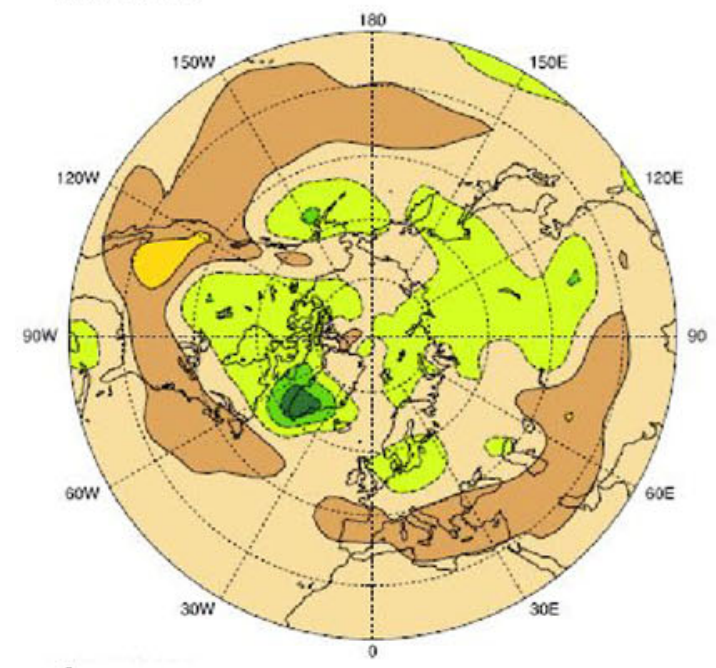

i

\section{CAM-ERA40}

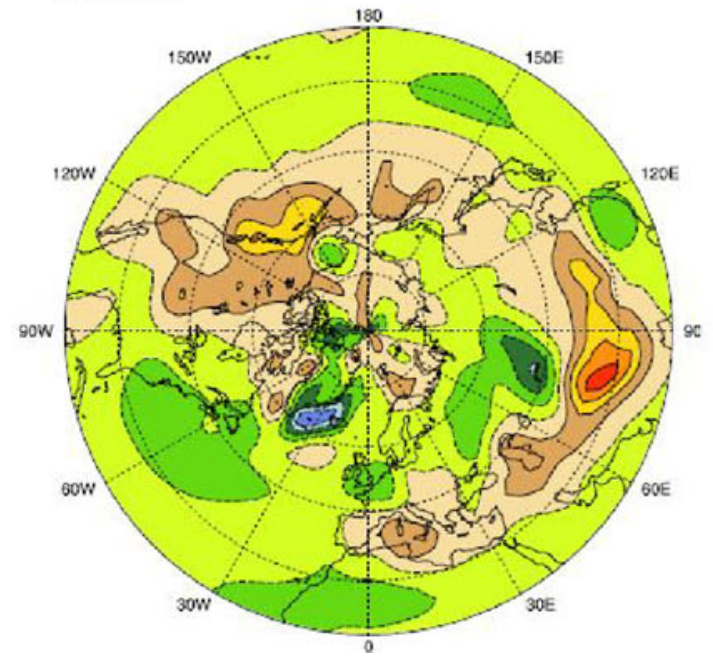

$\mathbf{j}_{\text {ERA-40 Fr }} \quad \sigma=0.7$

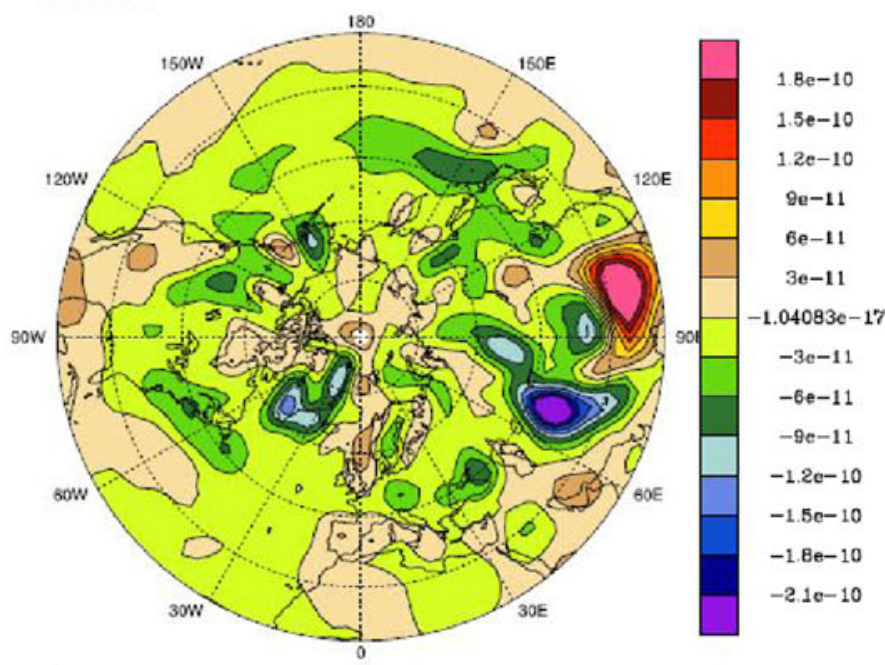

$\mathbf{k}$
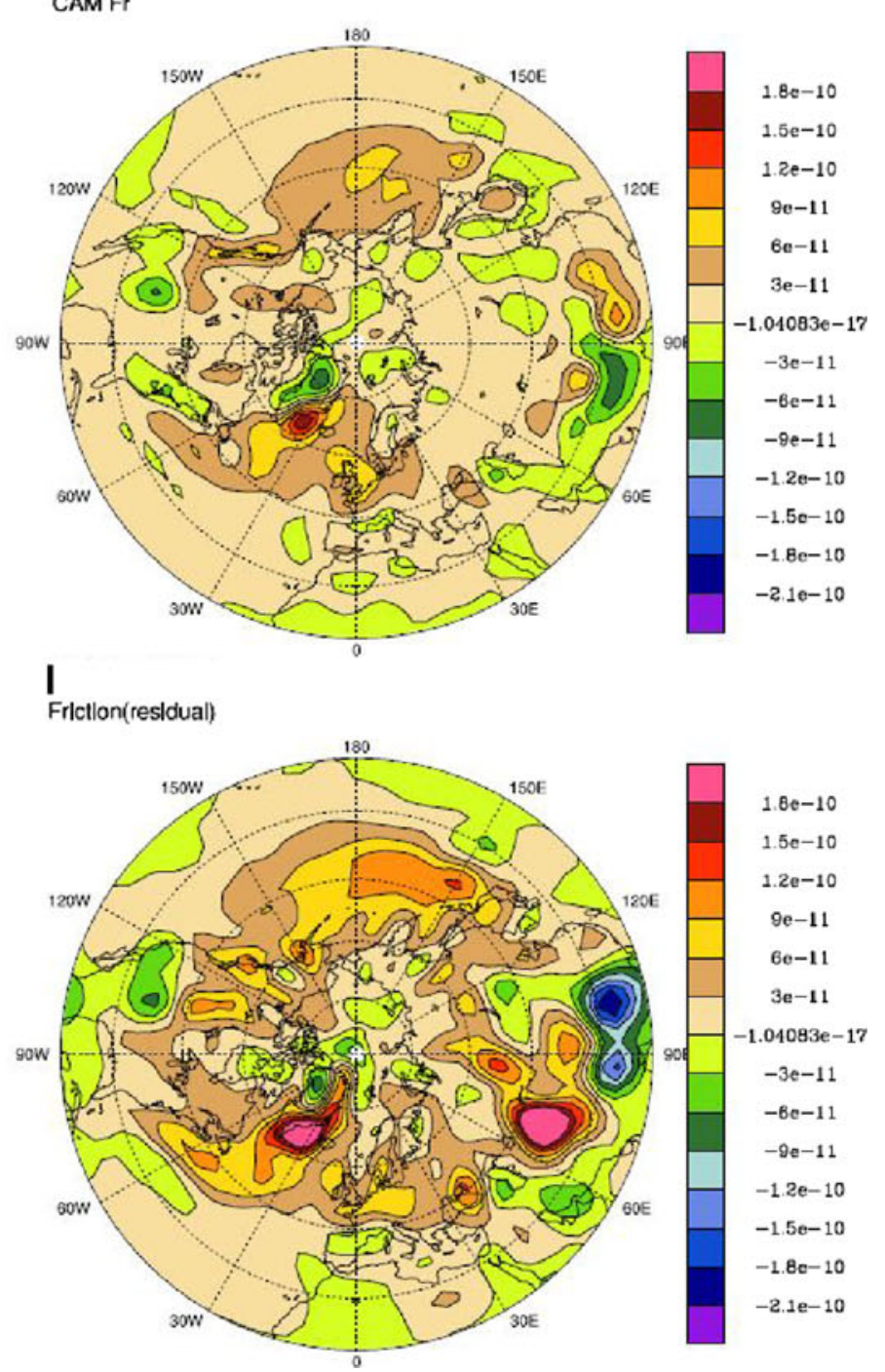

$1.8 \mathrm{e}-10$

$1.5 e-10$

$1.2 e-10$

$9 \mathrm{e}-11$

$6 \mathrm{e}-11$

$3 e-11$

$-1.04083 e-17$

$-3 e-11$

$-6 e-11$

$-9 e-11$

$-1.2 e-10$

$-1.5 e-10$

$-1.8 \mathrm{e}-10$

$-2.1 \mathrm{e}-10$

Fig. 5 continued 


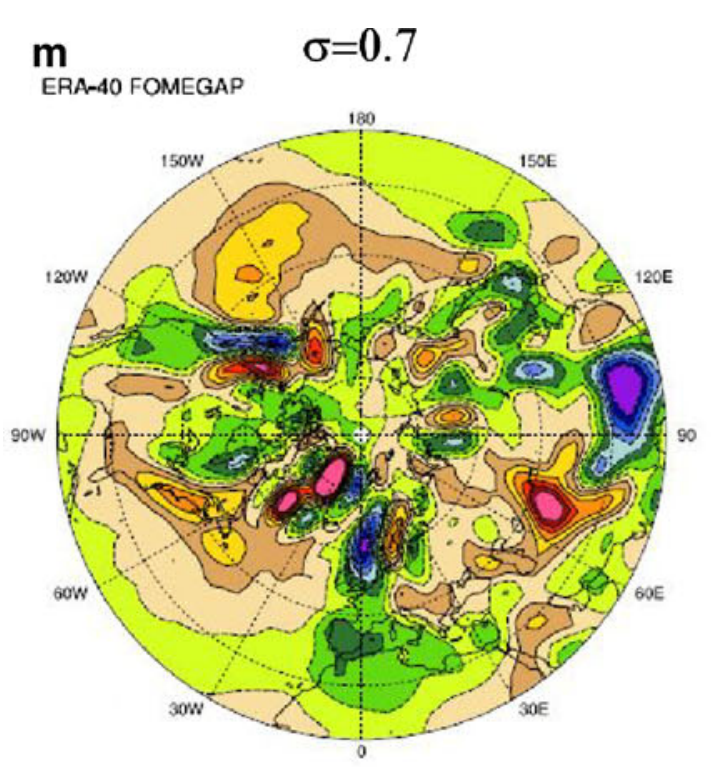

n

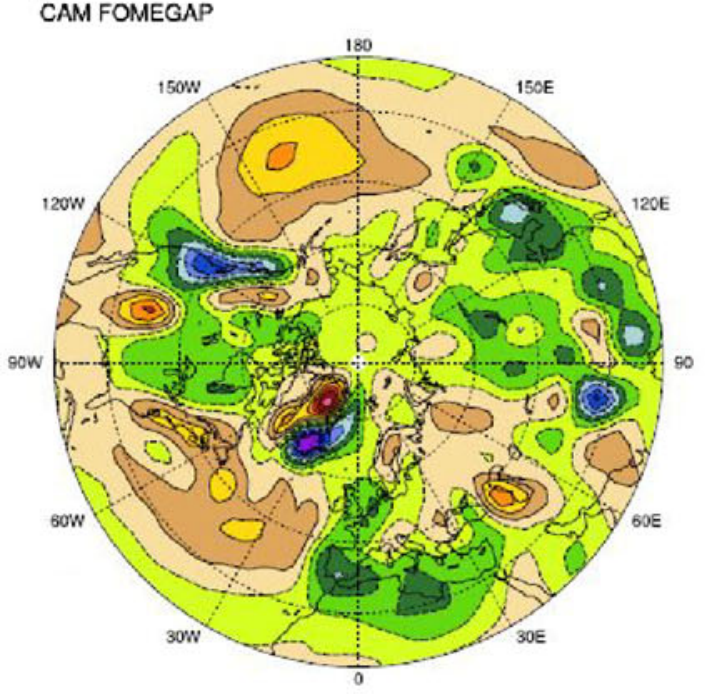

O

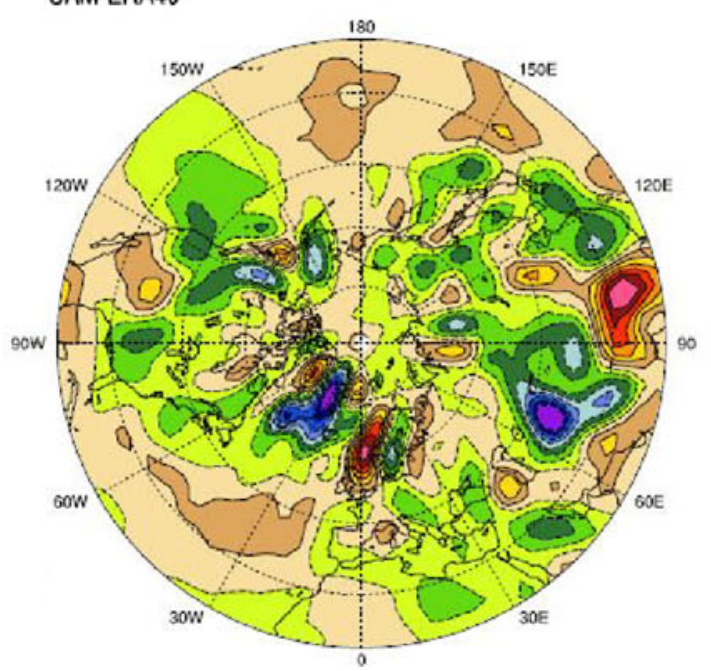

$\mathbf{p}_{\text {ERA-40 UZETAX }} \quad \sigma=0.7$

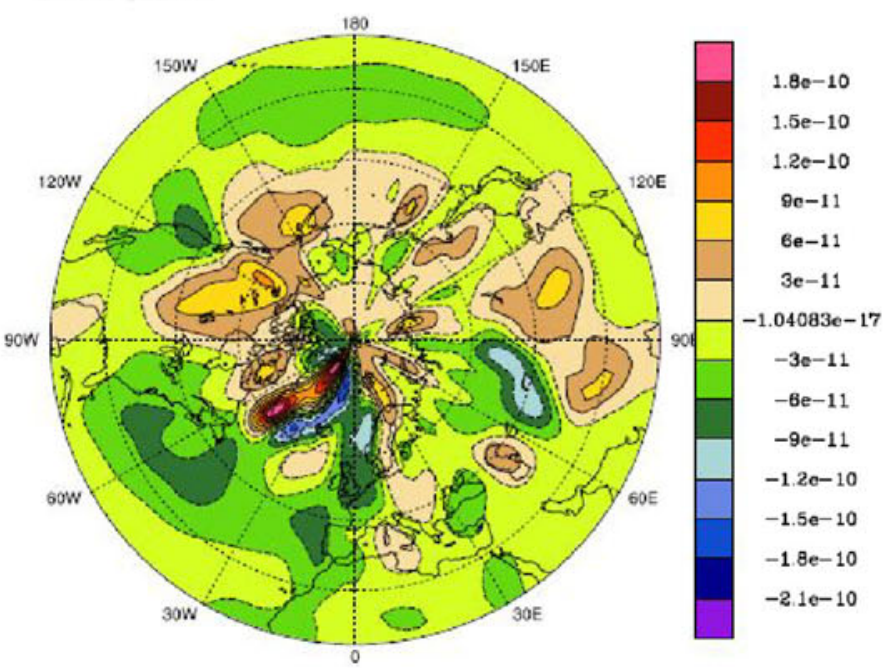

q

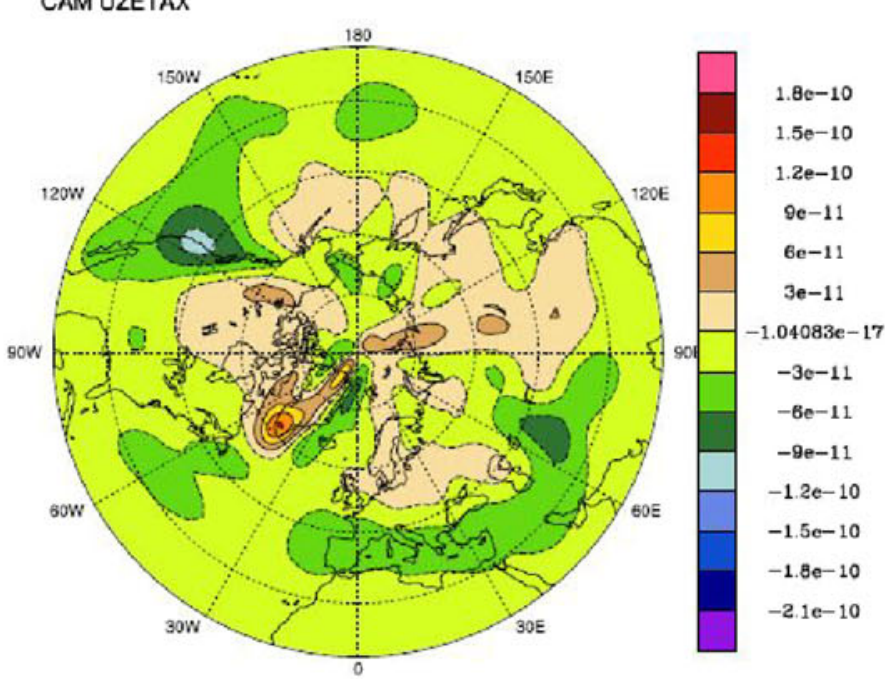

$r$

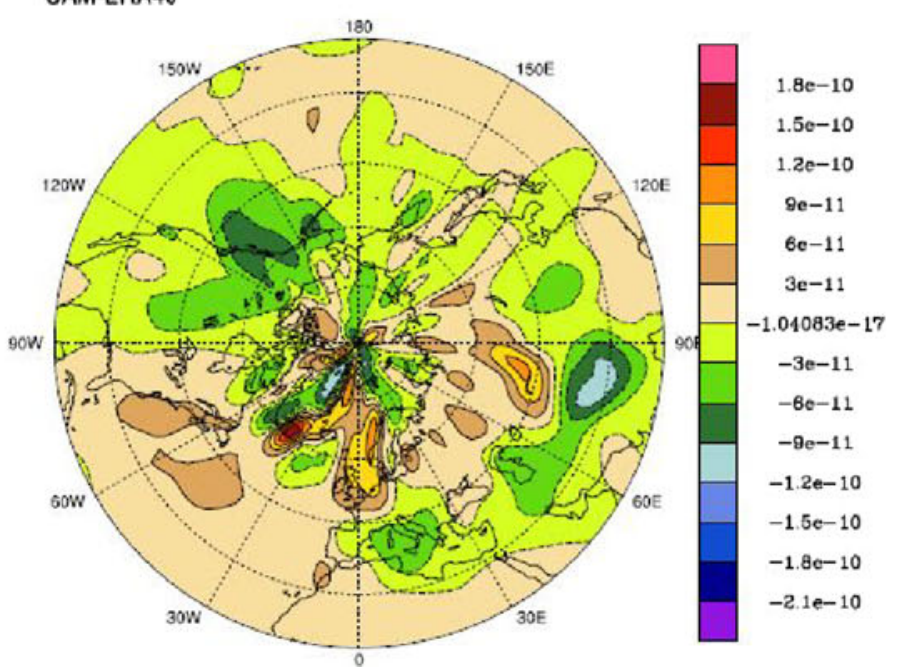

Fig. 5 continued 
Fig. 6 Vorticity equation terms grouped by contributions to the vorticity bias equation at two levels: left column representative of the upper troposphere, right column representative of the lower troposphere. Top row: terms that are linear combinations of the bias, second row: terms that are nonlinear in the bias, third row: transient contributions to the time mean, bottom row, remaining terms as needed for balance, implying primarily friction and diffusion. Units are $\mathrm{s}^{-2}$
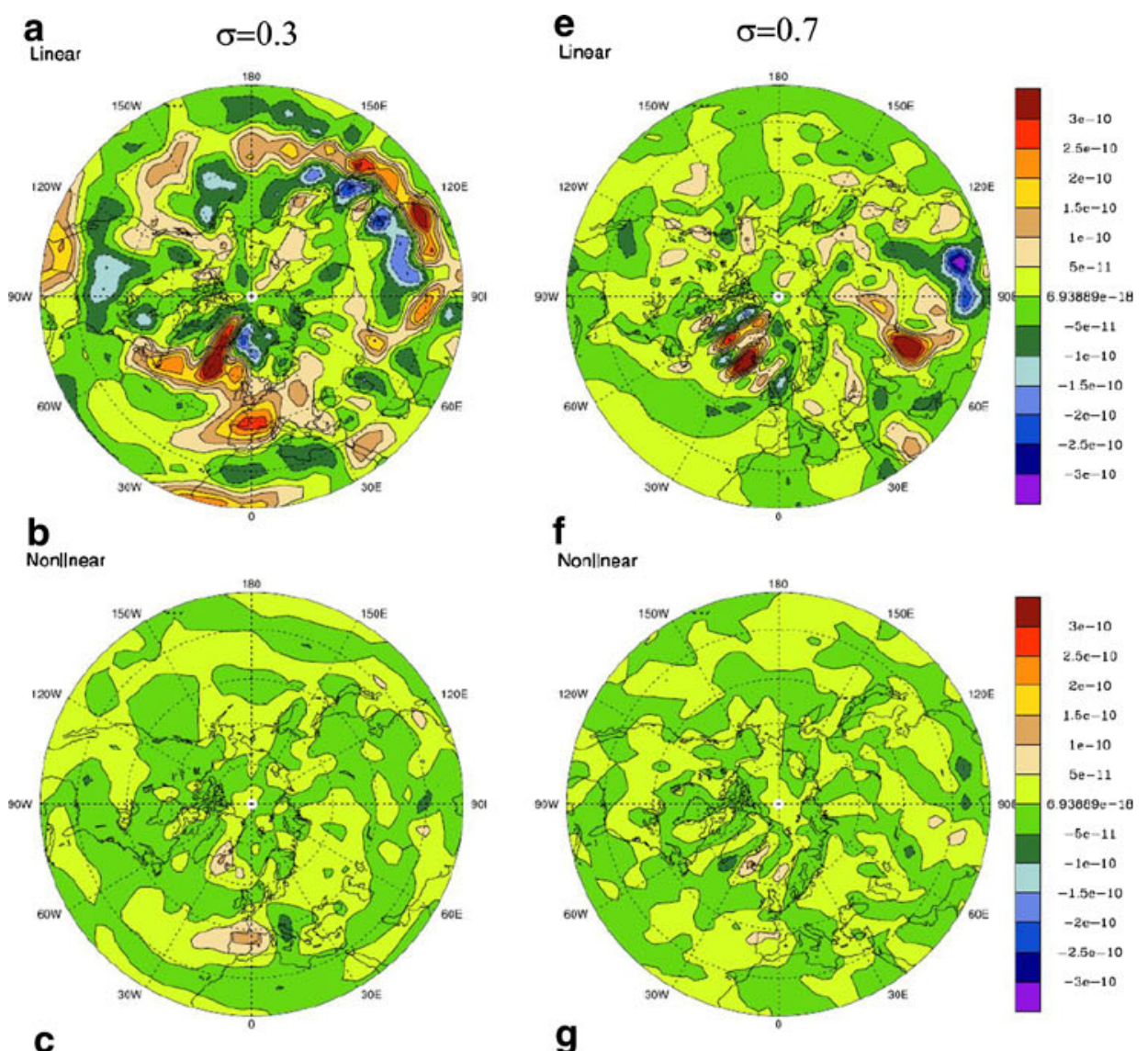

Transient

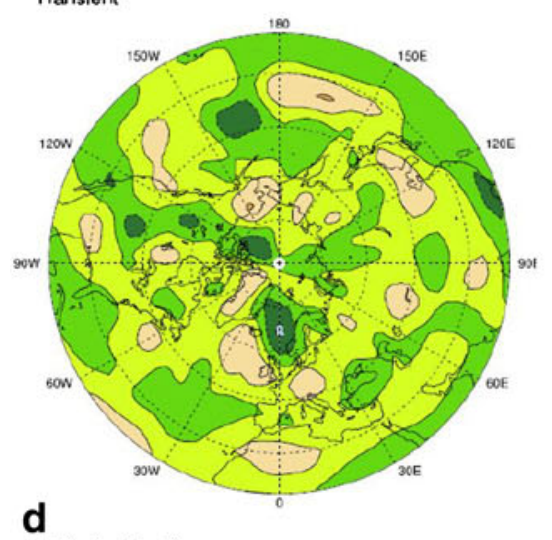

Friction(residual)

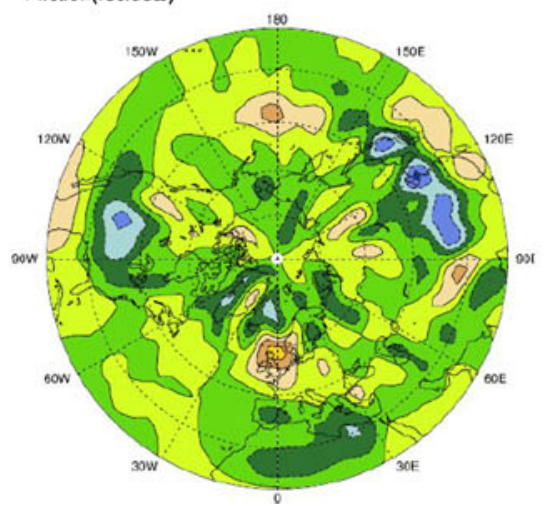

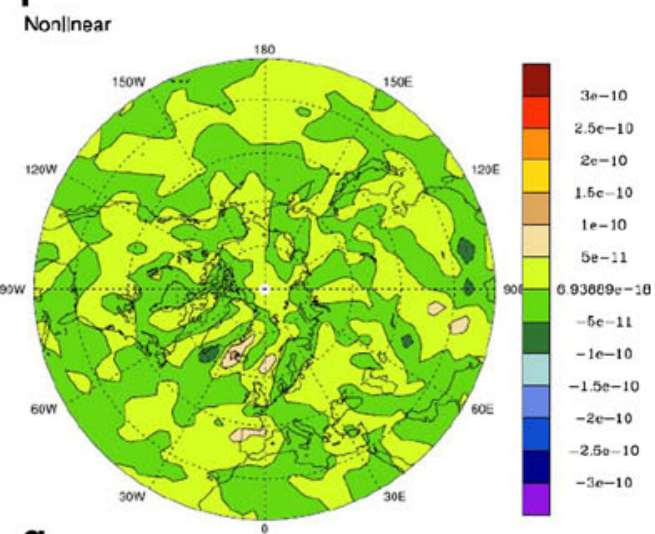

g

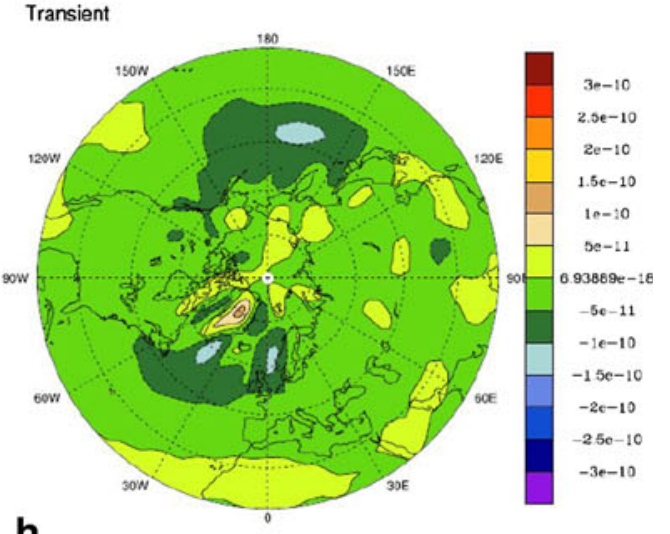

h

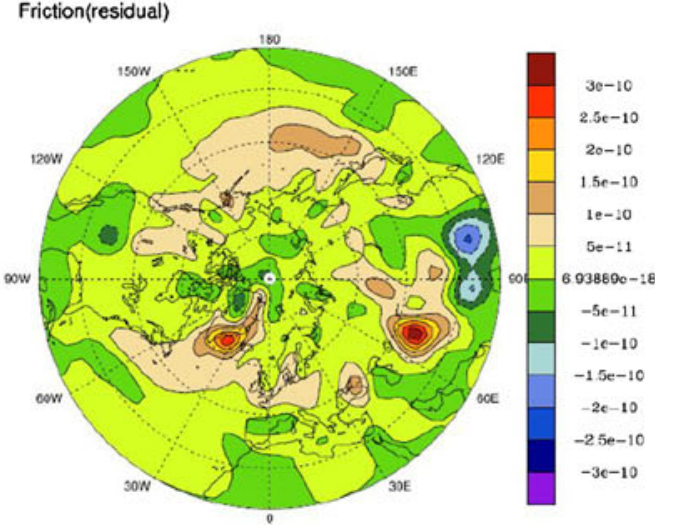


At the upper level $(\sigma=0.3)$ the linear terms are generally largest along the two storm tracks, and over the Arctic (away from topography). Along and upstream from the NPST, there is a curious elongated dipolar pattern that is coming mainly from the friction residual (Fig. 6d) and to a lesser degree from the zonal advection of relative vorticity (Fig. 4f). It is perhaps reasonable to imagine friction causing the dipolar pattern if the amplitude of shear vorticity is being reduced more strongly in CAM3; recall that Fig. 41 shows strong residual (presumably mainly friction, but possibly the other contributors to the residual mentioned above) in CAM3 along the East Asian subtropical jet that is not present in the ERA-40 data. Also recall that the East Asian subtropical jet (Fig. 3a-c) has small bias in the region, and the time mean zonal wind is positive. Part of the dipolar pattern is due to the positive trend in relative vorticity with latitude (from shear vorticity change) along the subtropical jet. However, the subtropical jet axis is oriented WSW to ENE and that northward component of the jet axis (as air moves downstream) creates a zonal gradient of vorticity as well. It is very difficult to see in $2 \mathrm{~g}, \mathrm{~h}$, but the zonal gradient of vorticity is negative over much of China and extending into the Pacific (from 20 to $35^{\circ} \mathrm{N}$ ). Similarly, the zonal gradient of vorticity is negative over northwest Africa (roughly $10-25^{\circ} \mathrm{N}$ ). In both locations the zonal advection term (Fig. $4 \mathrm{f}$ ) is therefore negative. These negative areas extend even further north due to the negative values contributed by transients to the zonal advection (not shown, but partially visible in Fig. 6c). Similarly, the northward component of the jet axis causes a positive vorticity gradient even further north of both those regions. The result is the dipole pattern in the zonal advection over China has opposite sign in CAM3 and ERA-40 resulting in the large bias (Fig. 4d-f). The vorticity patterns in CAM 3 and ERA-40 in Fig. $3 g$, $h$ are pretty similar however, the ERA-40 pattern is more 'noisy'. The discussion of storm tracks above mentioned that ERA-40 has generally larger amplitude in vorticity (and meridional wind) at generally all horizontal scales in middle latitudes; the greater amplitude in small horizontal scales is magnified by the derivatives of the vorticity gradient. So, the pattern of zonal advection bias has some large peak values. Hence, much of this dipolar pattern in the linear group of terms (Fig. 6a) near East Asia comes from greater friction in CAM3 and from small scales in ERA-40 zonal advection not present in CAM3.

Elsewhere, the linear group of terms has positive values near the dateline (30-45 N) that are captured by transients and friction and so result from an imbalance in linear terms, primarily a tilting term $(\partial \omega / \partial y \partial u / \partial p)$ bias and the quasi-geostrophic divergence $(f \partial \omega / \partial p<0$, Fig. 4i) bias. Further east and north the zonal advection bias (recall Fig. 4f) dominates the negative values $(45-60 \mathrm{~N}$;
150-180 W); while further east and south the positive values come from the meridional advection term $(v \partial \zeta / \partial y)$ bias (35-50 N; 130-110 W). Over North America, the quasi-geostrophic divergence term $(f \partial \omega / \partial p$; Fig. 4 i, with appropriate sign reversal) is a primary contributor to the linear terms at $\sigma=0.3$. At lower elevation over North America, friction (Figs. 51 or $6 \mathrm{~h}$ ) is a primary balance to the quasi-geostrophic divergence term (Fig. 5o, with reversed sign) in the linear terms (Fig. 6e). At the start of the NAST, the linear terms are positive over Atlantic Canada and Labrador Sea, mainly from the relative vorticity part of the divergence term $(\zeta \partial \omega / \partial p)$ whose positive values there are notably less in CAM3 consistent with the southward shift of the NAST in CAM3 relative to ERA-40 plus the weaker cyclones in CAM3 at the start of the NAST. The shift in the NAST at the start creates a dipole over the Atlantic in $\zeta \partial \omega / \partial p$ (not shown) that is visible even in Fig. 6a. Near the Greenwich meridian of the far north Atlantic is a dipole (negative north of positive) in the linear terms (Fig. 6a) that results from zonal advection bias dipole (partly cancelled by the meridional advection bias) and the quasi-geostrophic divergence $(f \partial \omega / \partial p<0)$ bias being displaced further south in CAM3. The linear terms Greenwich meridian dipole require friction for balance (Fig. 6d, again related to the NAST error, recall Fig. 4j, k) with a contribution from the transients (Fig. 6c) to the northern part $\left(60-75^{\circ} \mathrm{N}\right)$ of that dipole. Finally, the linear terms over southwestern Europe are large and positive from the quasi-geostrophic divergence $(f \partial \omega / \partial p<0)$ bias (Fig. 4i). The southward displacement in CAM3 of the downstream end of the NAST, and its impact on the subtropical jet across north Africa cause this $f \partial \omega / \partial p$ to be $<0$ in CAM3 but $>0$ in ERA-40 over France.

\section{Near-surface bias}

Near the surface, terms evaluated at $\sigma=0.95$ provide some insight into the sea level pressure bias (whose associated winds lead to surface wind and consequently sea ice biases). It is well established that a surface high pressure in a polar region can be associated with colder air (e.g. Petterssen 1956). That connection guides the interpretation below. The winter Arctic sea level pressure (SLP) patterns for ERA-40, CAM3, and their difference (bias) are plotted in Fig. 7a-c. The bias has generally lower pressure over the Arctic, with stronger low pressure at the Beaufort high and a relative high pressure in the Barents Sea.

The patterns for the two horizontal advection terms at a representative upper level $(\sigma=0.3)$ were shown in Fig. $4 \mathrm{a}-\mathrm{f}$. The terms have a lot of cancellation and the summation was discussed above. The summations of the two horizontal advection terms at $\sigma=0.95$ are shown in 


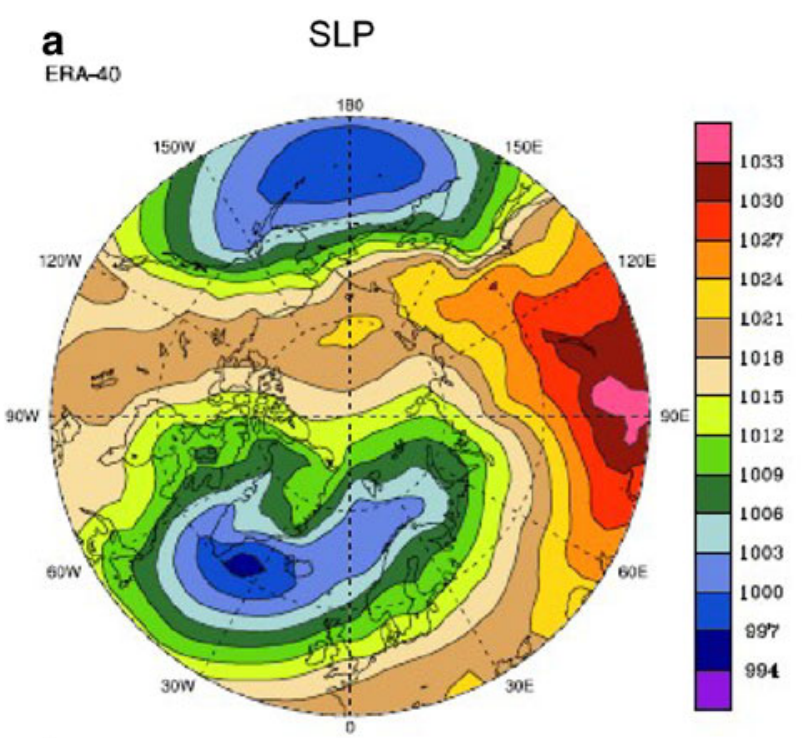

$\mathbf{b}_{\mathrm{CAM}}$

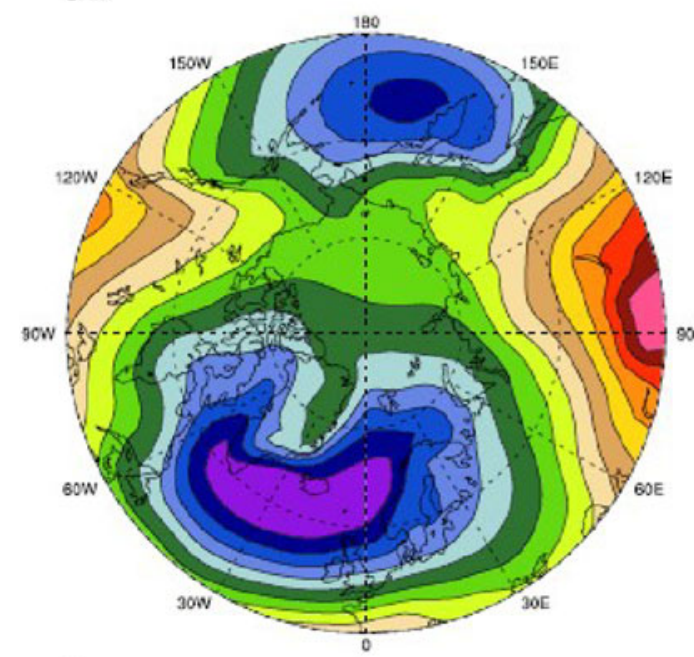

C

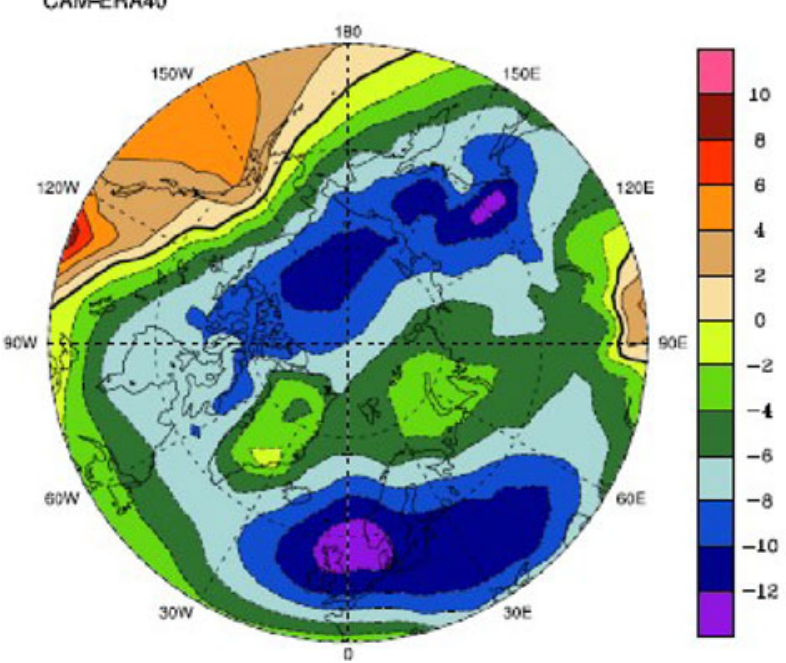

d $\quad \sigma=0.95$

ERA-40 UZETAX+VZETAY

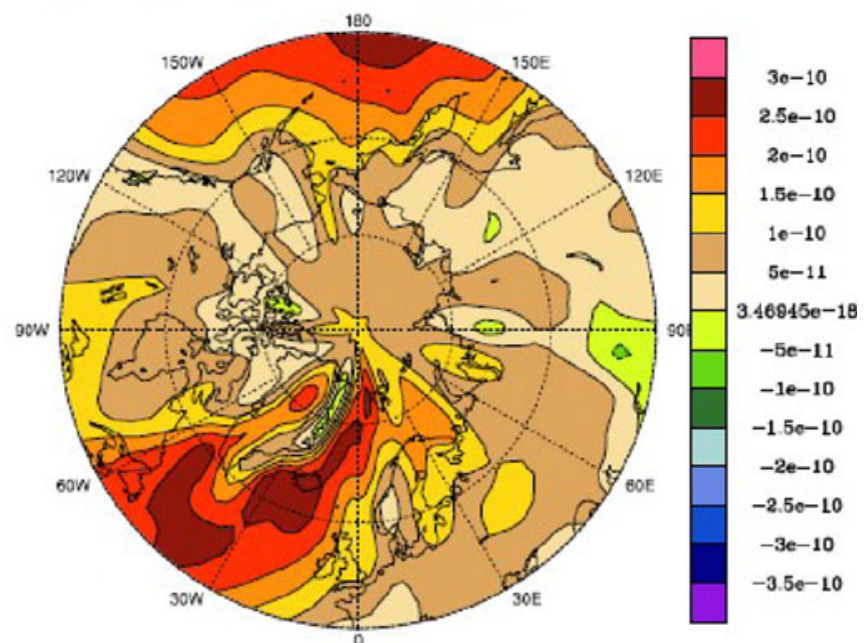

e

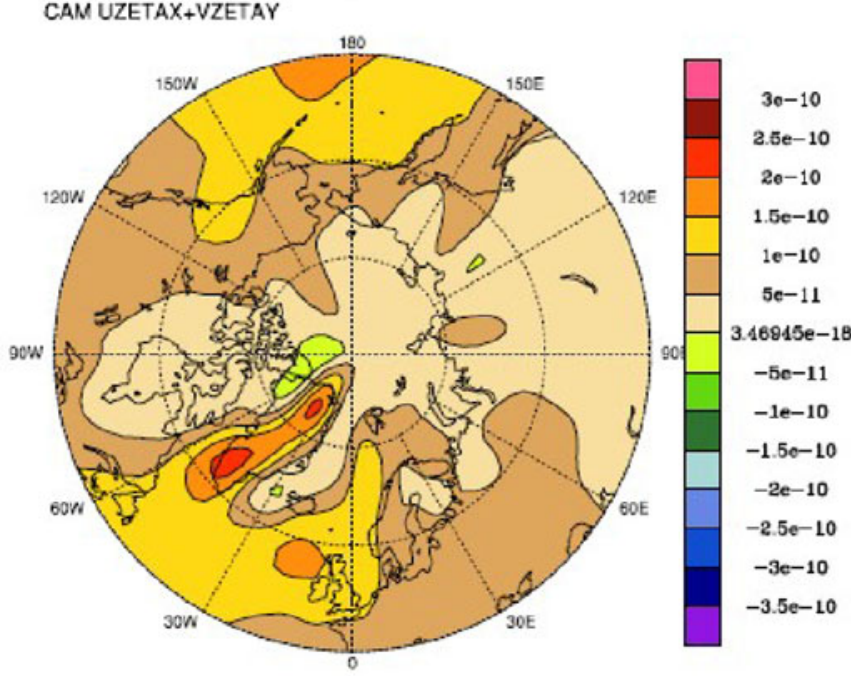

f

CAM-ERA40

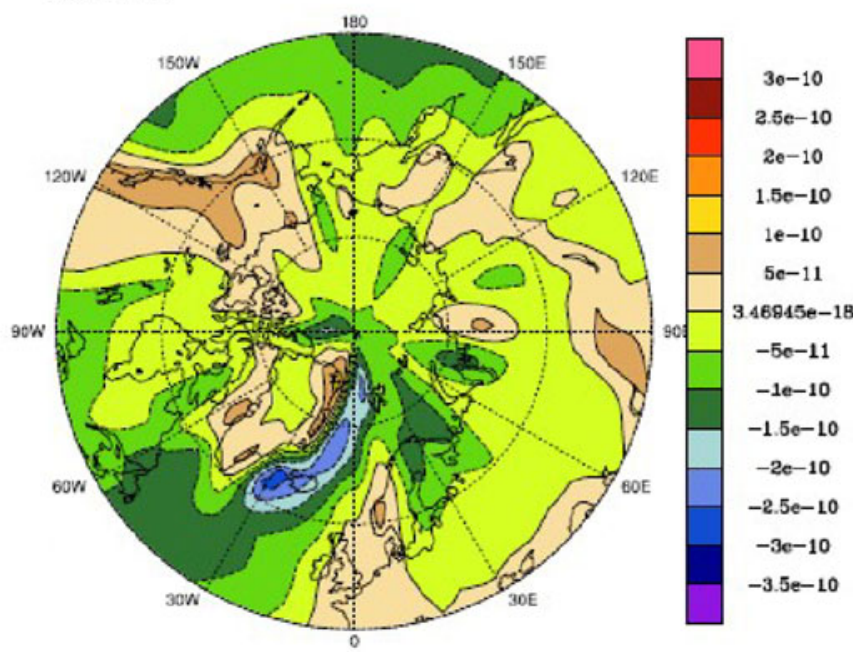


4 Fig. 7 a-c Sea level pressure (SLP) in hPa. d-f horizontal advection of vorticity $u \partial \zeta / \partial x+v \partial \zeta / \partial y$ at $\sigma=0.95$ in $\mathrm{s}^{-2}$. Top row ERA-40 data, middle row CAM3 data, bottom row bias. Horizontal vorticity advection is one of the primary drivers of the Beaufort high and its bias. Divergence terms vorticity tendency at $\sigma=0.95$. g-i The quasigeostrophic divergence term: $f \partial \omega / \partial p$ is strongly positive along the storm tracks but the NAST location error creates a strong anticyclonic vorticity bias tendency near the Barents and Kara Seas. To reveal details in other vorticity equation terms shown in Fig. 7, the same contour interval was used for panels $\mathbf{d}-\mathbf{l}$. $\mathbf{j}-\mathbf{l}$ The other divergence term: while the $\zeta \partial \omega / \partial p$ is positive along the storm tracks, the NAST location error also contributes negative vorticity bias tendency in the north Atlantic

Fig. 7d-f. The near-surface total horizontal advection is positive along and to the north of both storm tracks. The peak values are comparable to the peak values of the total horizontal advection at $\sigma=0.3$. Individually, the meridional advection places positive maxima along the first half (but north side) of the NPST and the first half of the NAST (for $v \partial \zeta / \partial y$ ) while for $u \partial \zeta / \partial x$, the positive values are further north and perhaps a little further east. Hence, at $\sigma=0.95$ the two terms no longer cancel over the NPST (though there is still some cancellation over the far north Atlantic). Both components of horizontal advection are more than twice as large in ERA-40 than CAM3 at this level, hence the bias is large and negative along and north of the NPST and NAST (Fig. 7f). The pattern of total advection bias for $\sigma=0.7$ (discernable from Fig. 5i, r) has generally smaller amplitude and is a mixture of the patterns described for the levels above and below.

Over the Beaufort Sea a SLP ridge is present in ERA-40 data (Fig. 7a) that is weaker (and without a peak value) in CAM3 data (Fig. 7b). This feature in the bias has been present in earlier versions of the NCAR general circulation models going back more than a decade. This feature in the bias has a westward tilt with increasing elevation; the minimum being near $150^{\circ} \mathrm{W}$ at the surface and $180^{\circ} \mathrm{W}$ at $500 \mathrm{hPa}$. Horizontal advection and divergence terms in the vorticity equation are prominent in this region near the surface. In ERA-40 data, cold air (shown in PGT) is advected northward out of central Russia, especially at longitudes near $90 \mathrm{E}$ as might be deduced from geostrophic motion using Fig. 7a. The horizontal advection has strong negative vorticity tendency (Fig. $7 \mathrm{~d}$, with sign reversed) over the Beaufort Sea. The quasi-geostrophic divergence term $(f \partial \omega / \partial p)$ also reinforces anticyclonic vorticity over the Beaufort (Fig. 7g). In CAM3, both the meridional and the zonal portions of this horizontal advection are weaker than in ERA-40 data, making the total horizontal advection (Fig. 7e) weaker than ERA-40 though the $f \partial \omega / \partial p$ is a bit stronger in CAM3. In addition, the divergence term in CAM3 data (Fig. 7h) opposes what vorticity tendency occurs from horizontal advection. The bias fields for the quasi-geostrophic terms (Fig. 7f, i) clearly create anticyclonic vorticity tendency poleward of $60 \mathrm{~N}$ along the dateline. Yet, the SLP bias (Fig. 7c) has cyclonic vorticity. Hence some more information is needed to understand this bias, namely the temperature bias above. The temperature and vorticity equations elements are brought together in the final section. Further to the east, along $150^{\circ} \mathrm{W}$ the horizontal advection terms (Fig. 7e) and divergence term (Fig. 7h) have opposite sign which seems consistent with the CAM3 pressure field being essentially flat from $60^{\circ} \mathrm{N}$ to the pole.

The SLP bias (Fig. 7c) also shows comparatively higher pressure (and associated negative vorticity) near the Barents Sea (e.g. near $75^{\circ} \mathrm{N}, 60^{\circ} \mathrm{E}$ ). This region of higher pressure is another feature seen for more than a decade in NCAR climate models. This feature has strong linkage to the NAST downstream track error. The divergence term is large and positive in the lower troposphere for developing lows (Grotjahn 1996). Positive values of the quasi-geostrophic divergence term are seen in Fig. $5 \mathrm{~m}, \mathrm{n}$ even though divergence is rather small at $\sigma=0.7$ level; Fig. 7g, h show the term near the surface at $\sigma=0.95$ and the positive values along the NAST are more prominent. On the downstream end of the NAST the relative vorticity increases so its contribution to the divergence term (Fig. $7 \mathrm{j}$, k) is also positive. From this understanding, the NAST downstream track error is quite easily seen in the quasigeostrophic divergence term (compare Fig. $7 \mathrm{~g}$, h). The NAST error is especially visible in the $\zeta \partial \omega / \partial p$ term (compare Fig. 7j, k) as is CAM3's weaker overall vorticity (making the term generally less in CAM3 data). The resultant bias in $(f+\zeta) \partial \omega / \partial p$ is quite large and negative over the Barents Sea and surroundings (Fig. 7i, 1) which is only partly counteracted by horizontal advection of vorticity as surface cyclones travel downstream (Fig. 7f, with sign reversal). CAM3's lack of contribution from the quasigeostrophic terms in the Barents Sea is a consequence of CAM3 tracking the cyclones well to the south, but it also causes the time averaged divergence term over the Barents Sea to have opposite sign from ERA-40 data (Fig. $7 \mathrm{~g}, \mathrm{~h}$ ). The sign reversal of this term causes a large bias.

\section{Further discussion and conclusions}

\subsection{Storm tracks}

The CAM3 bias in the northern hemisphere is dominated by errors in the handling of the two storm tracks. This is not too surprising since the vorticity is largest upstream and slightly north of these tracks (comparing Figs. 1 with $3 \mathrm{~g}-\mathrm{i}$ ). The error is dominated by the horizontal advection terms, though the two terms have quite a bit of cancellation. Despite the cancellation, biases in these individual terms 


\section{g}

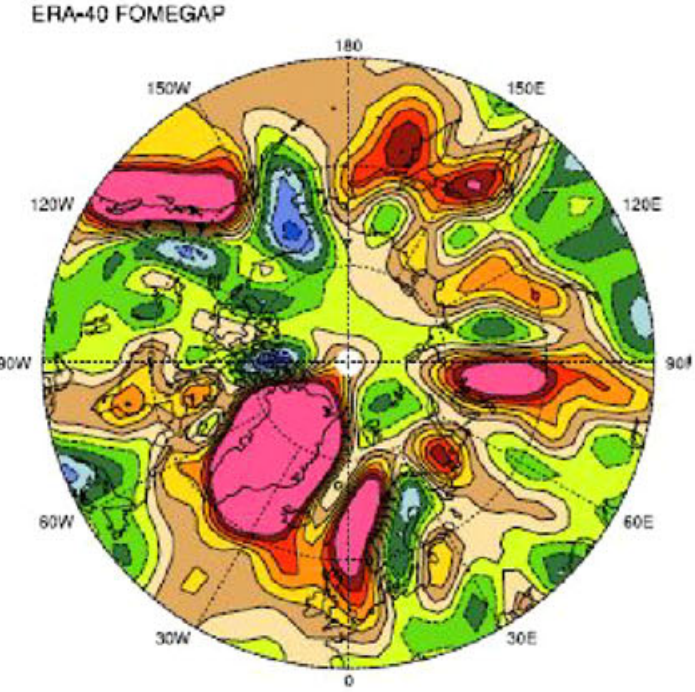

h

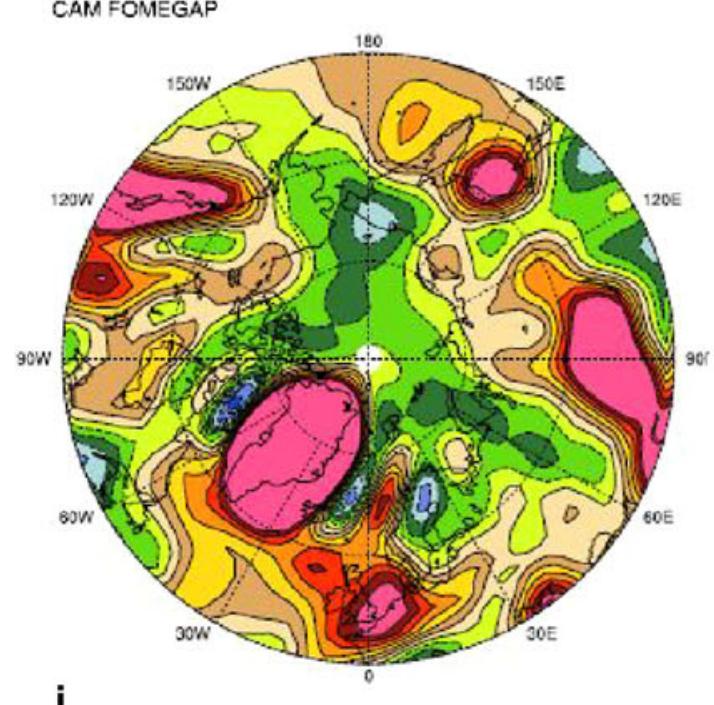

i

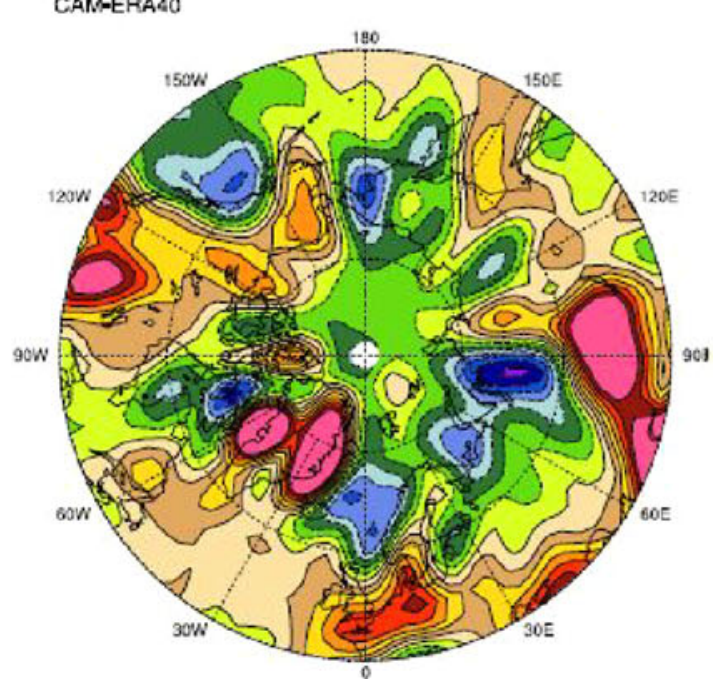

j $\quad \sigma=0.95$

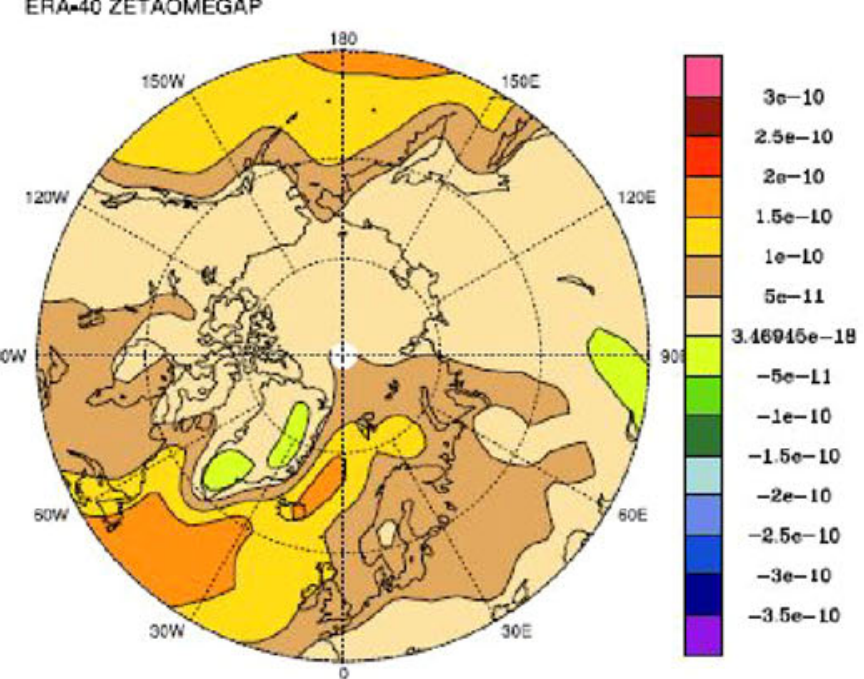

k

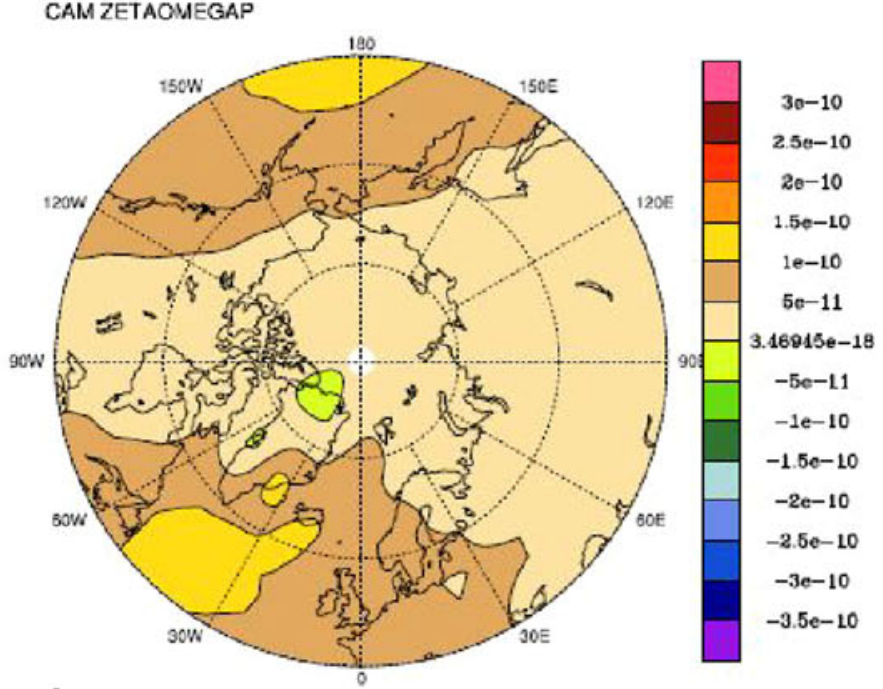

I

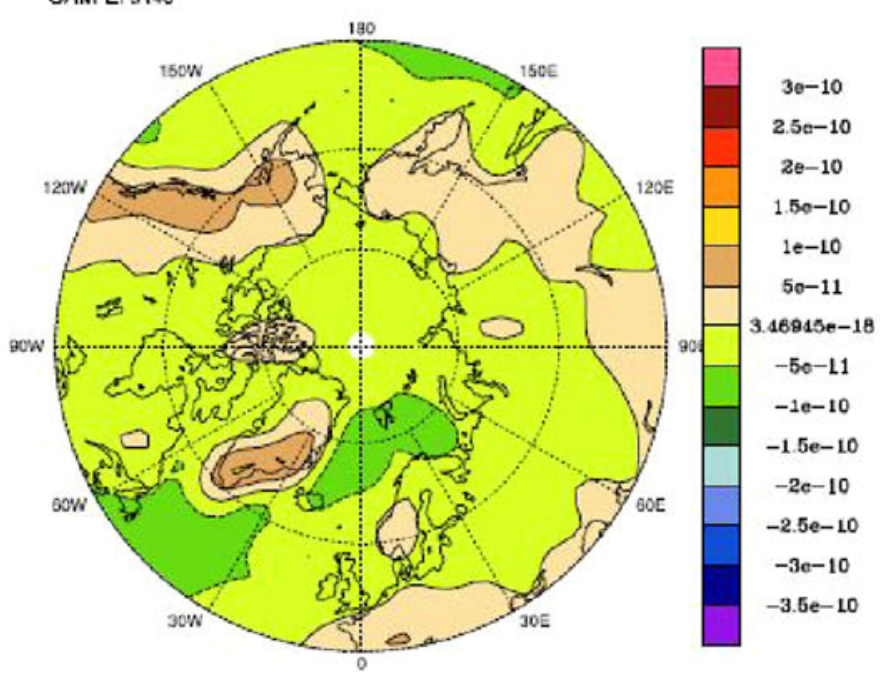

Fig. 7 continued 
along with the divergence term reappear when all the linear terms are combined. The transient terms bias reverses sign with height over the NPST and NAST because different terms rise to prominence: the vertical vorticity advection at low levels and the meridional advection at upper levels. It is also not surprising that the horizontal advection terms be prime contributors to the vorticity bias equation because measures of the storm tracks reveal two significant problems. First, measures of the storm strength (such as transient kinetic energy, transient enstrophy, and vorticity are much less in CAM3 even though the time mean jet streams are generally well simulated. In addition to amplitude, the track error, especially for the downstream end of the NAST is quite severe.

Another notable result is that the friction residual term is considerably stronger in CAM3 than in ERA-40 with the caveat that the term is a residual and numerical formulation issues may be significant contributors to the term. The bias of individual terms gives the impression that cyclogenesis (as measured by the far smaller storm track proxy values of Fig. 1) is being underachieved in CAM3 due to the divergence terms being notably weaker in CAM3. Hence the vorticity and enstrophy are smaller. It may be that the stronger friction is picking up some of the role that might be played by the eddies in drawing energy from the subtropical jets on the upstream ends of the storm tracks. This interpretation is consistent with the lower transient heat fluxes found by PGT at the start of each storm track. The reduced amplitude of the eddies may be a factor in the CAM3 storm track error on the downstream end, since larger eddies would deflect the flow more greatly. In addition, PGT also found that precipitation processes were much stronger in CAM3 which also may be filling a role in the energy balance that is not fully met by dry dynamics in CAM3 though the greater precipitation may arise because storms taking a more southerly track can tap more subtropical moisture (however, testing these ideas is beyond the scope of this project).

\subsection{Arctic region}

This work was motivated originally by concerns over the CAM3 biases in the Arctic surface climate. While the surface winds have been problematic for sea ice modeling, one can deduce gross features of the wind from the surface pressure and the surface pressure from analysis of the vorticity and temperature patterns.

This study identified sizable under-prediction of the enstrophy and kinetic energy over the Arctic region (Fig. 1c, f). Friction, as a residual in the calculation, at upper levels (Fig. 4k) had much stronger magnitude in CAM3 over several regions, including the Arctic. The Friction bias has a dipole (negative near $70^{\circ} \mathrm{N}$, positive near $55^{\circ} \mathrm{N}$ along $10^{\circ} \mathrm{W}$ ) with peak magnitudes around $1.5 \times 10^{-10} \mathrm{~s}^{-2}$ (Fig. 41). For developing frontal cyclones $\mathrm{f} \partial \omega / \partial p<0$ at upper levels (where there is upward motion peaking below) and acts to oppose the upper level advection (Grotjahn 1996). Figure 4g, h is largely negative along the NPST and NAST, with a tendency for larger values on the downstream end of each track. The quasi-geostrophic divergence $(f \partial \omega / \partial p<0)$ being displaced further south in CAM3 also contributes to the dipole (positive peak at $68 \mathrm{~N}$ and negative peak at $47^{\circ} \mathrm{N}$, along the Greenwich meridian; Fig. 4i). These friction and divergence terms have similar dipole location, strength, and opposite sign suggesting that $\mathrm{f} \partial \omega / \partial p$ is a primary contributor to the friction residual at upper levels along the NAST and NPST, and more so in CAM3 than in ERA-40 (Fig. 4g, h, j, k). In the far northern Atlantic Ocean the zonal advection bias is also large; the error in the downstream NAST location creates a dipole in this bias (Fig. 4f) that has strong poles: negative near $80 \mathrm{~N}$ and positive near $53^{\circ} \mathrm{N}$ along $20^{\circ} \mathrm{E}$; however, the meridional advection (Fig. 4c) has a very similar pattern and opposite sign so those large terms nearly cancel. The pattern of the meridional advection is stronger and determines the bias pattern for the total horizontal advection over Europe and the North Sea (and similarly along the NPST).

One of two prominent features of the SLP bias over the Arctic (Fig. 7c) is the negative bias where the Beaufort high is located because that high is much too weak in CAM3. To understand this feature in the bias one must consider both the forcing of vorticity at low levels as well as the temperature bias above. As mentioned above, colder regions generally have shallow relatively high pressure while warmer regions have a shallow surface low. Temperature equation features were discussed extensively in PGT and elements of that discussion are included here. Figure 8 illustrates schematically the main features described above near the Beaufort high by following a few key variables along the $150^{\circ} \mathrm{W}$ meridian. Numeric values are representative values found in the biases; some of which are shown in PGT. Other numbers and the geopotential height curves are based on data accessible from the NCAR-maintained CAM3 homepage. Through much of the lower troposphere north of the Beaufort high CAM3 has a warm bias ( $\mathrm{Tb}$ values in Fig. 8) which causes a general lowering of the SLP over most of the Arctic compared to ERA-40 data. Hence the SLP bias is generally negative over most of the Arctic (Fig. 7c). Figure 8 also indicates that low level meridional heat flux (divergence) is stronger in CAM3 than ERA-40 supporting cooling temperature tendency (near $65 \mathrm{~N}$ ) and thus cold bias at low elevations over Alaska. Over the Beaufort Sea, the low level divergence term supports anticyclonic (negative) vorticity tendency in CAM3 and ERA-40 but the meridional distribution differs (being weaker in CAM3 where the 
Beaufort high should be centered) and thus the bias supports cyclonic vorticity tendency near $70 \mathrm{~N}$. The low level total horizontal advection supports anticyclonic (negative) vorticity tendency, but more strongly in ERA-40. Hence, the overall warm bias supports lower SLP while the quasigeostrophic vorticity tendency terms (divergence plus total horizontal advection) are more effective at building anticyclone vorticity in ERA-40. The result is a strong SLP low bias centered where the Beaufort High should be.

The second area of interest is the Barents Sea and Novaya Zemlya Islands region where the SLP bias (Fig. 7c) has a relative maximum. As with the under-predicted Beaufort High, this SLP bias is a long standing problem seen in earlier and current versions of the NCAR models. That region occupies the middle of the schematic diagram (Fig. 9) which shows key elements along 60E. The temperatures are especially elevated in CAM3 on the poleward side of the SLP relative maximum and over much of the middle and lower troposphere shown in the figure. But where the SLP bias has its weak relative maximum, CAM3 has slightly colder temperatures, consistent with expectations from hydrostatic balance. The relatively higher pressure is equivalent barotropic due to its colocation with a CAM3 warm bias in the middle and lower

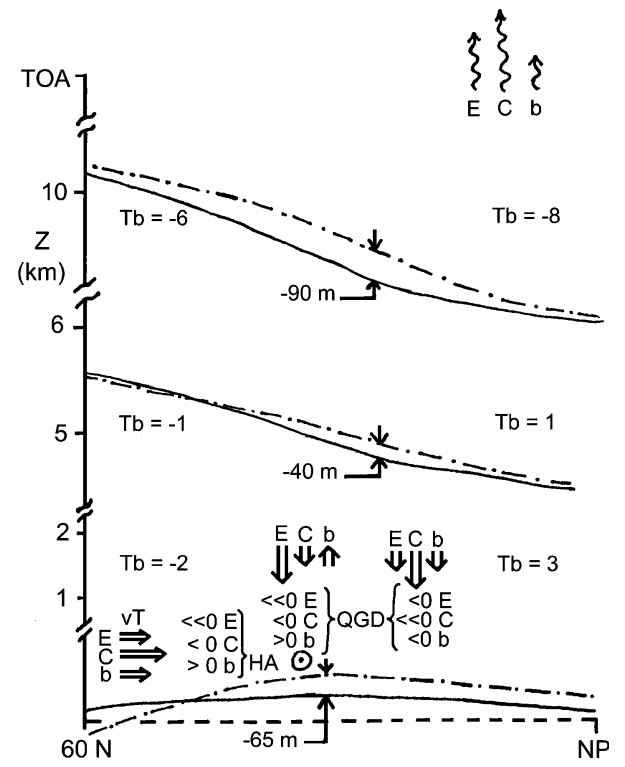

Fig. 8 Schematic diagram of factors that create the bias near the Beaufort high along $150 \mathrm{~W}$. Schematic surfaces of 200, 500, and $1,000 \mathrm{hPa}$ geopotential height are shown where CAM3 data are solid lines and ERA-40 data are dot-dashed lines. Tb refers to temperature bias. $E, C$, and $b$ refer to ERA-40, CAM3, and bias quantities. vT is meridional wind times temperature over a seasonal average. HA refers to the horizontal advection of relative vorticity contribution to vorticity tendency: $\mathrm{HA}=-u \partial \zeta / \partial x-v \partial \zeta / \partial y$. QGD refers to the quasigeostrophic divergence term: $f \partial \omega / \partial p$ which can be deduced from the vertical motion (double-shafted vertical arrows). Wavy lines at top of atmosphere (TOA) are net radiative cooling. Dashed line indicates horizontal

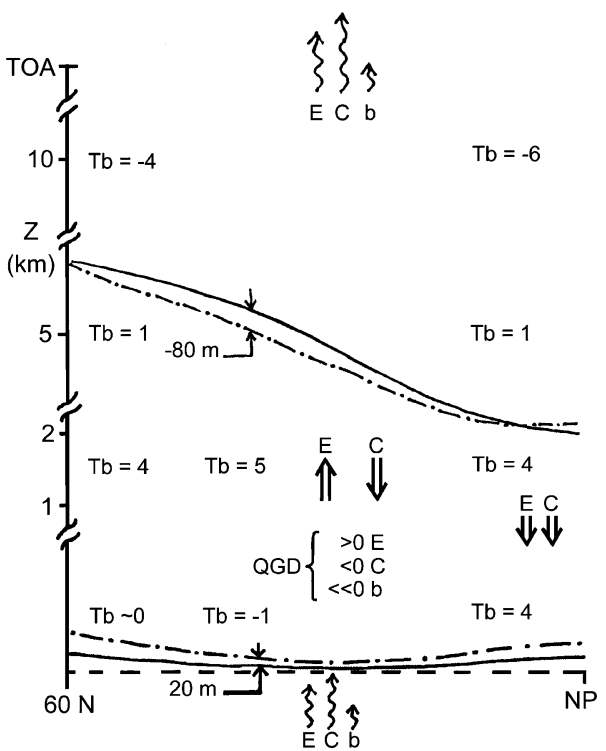

Fig. 9 Similar to Fig. 8 except showing factors that create the bias near the Barents Sea along 60E. Schematic surfaces of 500 and $1,000 \mathrm{hPa}$ are plotted using CAM3 data (solid lines) and ERA-40 data (dot-dashed lines). $\mathrm{Tb}$ is bias temperature. $\mathrm{E}, \mathrm{C}$, and $\mathrm{b}$ refer to ERA40, CAM3, and bias data. QGD is quasi-geostrophic divergence term vorticity tendency: $f \partial \omega / \partial p$. Wavy lines show TOA net radiation and bottom surface sensible heat flux

troposphere (Fig. 9). The divergence term plays a prominent role in this bias. Along the NAST the divergence term is generally positive (Fig. $7 \mathrm{~g}, \mathrm{~h}, \mathrm{j}, \mathrm{k}$ ) with the non-quasigeostrophic part $(\zeta \partial \omega / \partial p)$ being positive on the downstream end of the storm track. The ERA-40 NAST ends in the Barents Sea (QGD $>0$ in Fig. 9) while the CAM3 track is much further south (leaving QGD $<0$ over the Barents in CAM3 and in the bias). Hence due to the NAST location error, the divergence term is contributing anticyclonic (negative) vorticity tendency (and thus higher SLP) in an environment of generally warmer temperatures (which reduce SLP). Finally, the connection between the Barents Sea SLP bias and the NAST is consistent with the 1-pt correlation analysis of reanalysis data shown in PGT. PGT found that higher precipitation over England and France (due to frontal cyclones taking a more southerly track) are correlated with higher SLP over Novaya Zemlya a day or two later. Further analysis, specifically to understand what causes these temperature biases and the downstream NAST location error are beyond the scope of this vorticity bias equation study.

Acknowledgments This work was supported by NSF grant ATM 0354545. ECMWF ERA-40 data used in this study/project were provided by ECMWF from the main data server. The authors thank the members of NCAR's Climate Modeling Section, Computer Software and Engineering Group, and Scientific Computing Division for their contributions to the development of CAM3. We thank Dr. Grant Branstator for suggesting that we study a vorticity bias 
equation. The authors also thank the helpful advice from two anonymous reviewers.

Open Access This article is distributed under the terms of the Creative Commons Attribution Noncommercial License which permits any noncommercial use, distribution, and reproduction in any medium, provided the original author(s) and source are credited.

\section{References}

Branstator G (1990) Low-frequency patterns induced by stationary waves. J Atmos Sci 47:2184-2198. doi:10.1175/1520-0469 (1990)047<0629:LFPIBS >2.0.CO;2

Carlson TN (1991) Mid-latitude weather systems. Routledge, London, $507 \mathrm{pp}$

Collins WD, Rasch PJ, Boville BA, Hack JJ, McCaa JR, Williamson DL, Kiehl JT, Briegleb B, Bitz C, Lin SJ, Zhang M, Dai Y (2004) Description of the NCAR community atmosphere model (CAM3). Tech. Rep. NCAR/TN-464 STR. National Center for Atmospheric Research, Boulder, CO, 226 pp

DeWeaver E, Bitz CM (2006) Atmospheric circulation and its effect on Arctic sea ice in CCSM3 simulations at medium and high resolution. J Climate 19:2415-2432

Grotjahn R (1993) Global atmospheric circulations: observations and theories. Oxford, New York, 430 pp
Grotjahn R (1996) Vorticity equation terms for extratropical cyclones. Mon Wea Rev 124:2843-2858

Grotjahn R, Castello C (2000) A study of frontal cyclone surface and $300-\mathrm{hPa}$ geostrophic kinetic energy distribution and scale change. Mon Wea Rev 128:2865-2874

Hack JJ, Caron JM, Danabasoglu G, Oleson K, Bitz CM (2006) CCSM3-CAM3 climate simulation sensitivity to increased horizontal resolution. J Climate 19:2267-2289

Pan LL, Jin FF, Watanabe M (2006) Dynamics of synoptic eddy and low-frequency flow (SELF) interaction. Part III: Baroclinic model results. J Atmos Sci 63:1709-1725

Pan LL, Grotjahn R, Tribbia J (2010) Sources of CAM3 temperature bias during northern winter from diagnostic study of the temperature bias equation. Clim Dyn 35:1411-1427. doi: 10.1007/s00382-009-0608-6

Petterssen S (1956) Weather analysis and forecasting, 2nd edn, vol 1, Motion and motion systems. McGraw-Hill, New York, 428 pp

Uppala SM, Kållberg PW, Simmons AJ, Andrae U, da Costa Bechtold V, Fiorino M, Gibson JK, Haseler J, Hernandez A, Kelly GA, Li X, Onogi K, Saarinen S, Sokka N, Allan RP, Andersson E, Arpe K, Balmaseda MA, Beljaars ACM, van de Berg L, Bidlot J, Bormann N, Caires S, Chevallier F, Dethof A, Dragosavac M, Fisher M, Fuentes M, Hagemann S, Hólm E, Hoskins BJ, Isaksen L, Janssen PAEM, Jenne R, McNally AP, Mahfouf JF, Morcrette JJ, Rayner NA, Saunders RW, Simon P, Sterl A, Trenberth KE, Untch A, Vasiljevic D, Viterbo P, Woollen J (2005) The ERA-40 re-analysis. Quart J R Meteorol Soc 131:2961-3012 